\title{
COMPUTATIONAL MODELING OF THE FORWARD AND REVERSE PROBLEMS IN INSTRUMENTED SHARP INDENTATION
}

\author{
M. DAO, N. CHOLLACOOP, K. J. VAN VLIET, T. A. VENKATESH and S. SURESH $\dagger$ \\ Department of Materials Science and Engineering, Massachusetts Institute of Technology, Cambridge,
} MA 02139, USA

( Received 23 April 2001; received in revised form 6 August 2001; accepted 7 August 2001)

\begin{abstract}
A comprehensive computational study was undertaken to identify the extent to which elastoplastic properties of ductile materials could be determined from instrumented sharp indentation and to quantify the sensitivity of such extracted properties to variations in the measured indentation data. Large deformation finite element computations were carried out for 76 different combinations of elasto-plastic properties that encompass the wide range of parameters commonly found in pure and alloyed engineering metals: Young's modulus, $E$, was varied from 10 to $210 \mathrm{GPa}$, yield strength, $\sigma_{\mathrm{y}}$, from 30 to $3000 \mathrm{MPa}$, and strain hardening exponent, $n$, from 0 to 0.5 , and the Poisson's ratio, $v$, was fixed at 0.3 . Using dimensional analysis, a new set of dimensionless functions were constructed to characterize instrumented sharp indentation. From these functions and elasto-plastic finite element computations, analytical expressions were derived to relate indentation data to elasto-plastic properties. Forward and reverse analysis algorithms were thus established; the forward algorithms allow for the calculation of a unique indentation response for a given set of elasto-plastic properties, whereas the reverse algorithms enable the extraction of elasto-plastic properties from a given set of indentation data. A representative plastic strain $\varepsilon_{\mathrm{r}}$ was identified as a strain level which allows for the construction of a dimensionless description of indentation loading response, independent of strain hardening exponent $n$. The proposed reverse analysis provides a unique solution of the reduced Young's modulus $E^{*}$, a representative stress $\sigma_{\mathrm{r}}$, and the hardness $p_{\text {ave. }}$. These values are somewhat sensitive to the experimental scatter and/or error commonly seen in instrumented indentation. With this information, values of $\sigma_{\mathrm{y}}$ and $n$ can be determined for the majority of cases considered here, provided that the assumption of power law hardening adequately represents the full uniaxial stress-strain response. These plastic properties, however, are very strongly influenced by even small variations in the parameters extracted from instrumented indentation experiments. Comprehensive sensitivity analyses were carried out for both forward and reverse algorithms, and the computational results were compared with experimental data for two materials. (C) 2001 Acta Materialia Inc. Published by Elsevier Science Ltd. All rights reserved.
\end{abstract}

Keywords: Indentation; Mechanical properties; Finite element simulation; Large deformation; Representative strain

\section{INTRODUCTION}

The mechanical characterization of materials has long been represented by their hardness values [1, 2]. Recent technological advances have led to the general availability of depth-sensing instrumented micro- and nanoindentation experiments (e.g. [1-14]). Nanoindenters provide accurate measurements of the continuous variation of indentation load $P$ down to $\mu \mathrm{N}$, as a function of the indentation depth $h$ down to $\mathrm{nm}$. Experimental investigations of indentation have been conducted on many material systems to extract hard-

$\dagger$ To whom all correspondence should be addressed. Tel.: +1-617-253-3320; Fax: +1-617-253-0868.

E-mail address: ssuresh@mit.edu (S. Suresh) ness and other mechanical properties and/or residual stresses (e.g., [3-5, 9, 13-17], among many others).

Concurrently, comprehensive theoretical and computational studies have emerged to elucidate the contact mechanics and deformation mechanisms in order to systematically extract material properties from $P$ versus $h$ curves obtained from instrumented indentation (e.g., [3, 5, 11, 12, 16-21]). For example, the hardness and Young's modulus can be obtained from the maximum load and the initial unloading slope using the methods suggested by Oliver and Pharr [5] or Doerner and Nix [3]. The elastic and plastic properties may be computed through a procedure proposed by Giannakopoulos and Suresh [20], and the residual stresses may be extracted by the method of Suresh and Giannakopoulos [22]. Thin film systems have also been studied using finite element computations [23-25]. 
Using the concept of self-similarity, simple but general results of elasto-plastic indentation response have been obtained. To this end, Hill et al. [26] developed a self-similar solution for the plastic indentation of a power law plastic material under spherical indentation, where Meyer's law $\dagger$ was given a rigorous theoretical basis. Later, for an elasto-plastic material, self-similar approximations of sharp (i.e., Berkovich and Vickers) indentation were computationally obtained by Giannakopoulos et al. [18] and Larsson et al. [27]. More recently, scaling functions were applied to study bulk [11, 12, 19] and coated material systems [25]. Kick's Law (i.e., $P=C h^{2}$ during loading, where loading curvature $C$ is a material constant) was found to be a natural outcome of the dimensional analysis of sharp indentation (e.g., [11]).

Despite these advances, several fundamental issues remain that require further examination:

1. A set of analytical functions, which takes into account the pile-up/sink-in effects and the large deformation characteristics of the indentation, needs to be established in order to avoid detailed FEM computations after each indentation test. These functions can be used to accurately predict the indentation response from a given set of elastoplastic properties (forward algorithms), and to extract the elasto-plastic properties from a given set of indentation data (reverse algorithms). Giannakopoulos, Larsson and Vestergaard [18] and later Giannakopoulos and Suresh [20] proposed a comprehensive analytical framework to extract elasto-plastic properties from a single set of $P-$ $h$ data. Their results, as will be shown later in this study, were formulated using mainly small deformation FEM results (although they performed a number of large deformation computations). Cheng and Cheng [11, 12, 19], using an included apex angle of the indenter of $68^{\circ}$, proposed a set of universal dimensionless functions based on large deformation FEM computations, but did not establish a full set of closed-form analytical functions.

2. Under what conditions and/or assumptions can we extract a single set of elasto-plastic properties from a single $P-h$ curve with reasonable accuracy? Cheng and Cheng [19] and Venkatesh et al. [21] discussed this issue. However, without an accurate analytical framework based on large deformation theory, this issue cannot be addressed.

3. What are the similarities and differences between the large and small deformation-based analytical formulations? Chaudhri [28] estimated that equivalent strains of $25-36 \%$ were present in the

\footnotetext{
$\dagger$ Meyer's law for spherical indentation states that $P=\frac{K a^{m}}{D^{m-2}}$, where $m$ is a hardening factor, $D$ is the indenter's diameter, $a$ is the contact radius of the indenter, and $K$ is a material constant.
}

indented specimen near the tip of the Vickers indenter. These experimentally observed large strains justify the need for large deformation based theories in modeling instrumented sharp indentation tests.

In this paper, these issues will be addressed within the context of sharp indentation and continuum analysis.

\section{THEORETICAL AND COMPUTATIONAL CONSIDERATIONS}

\subsection{Problem formulation and associated nomencla- ture}

Figure 1 shows the typical $P-h$ response of an elasto-plastic material to sharp indentation. During loading, the response generally follows the relation described by Kick's Law,

$$
P=C h^{2}
$$

where $C$ is the loading curvature. The average contact pressure, $p_{\text {ave }}=\frac{P_{\mathrm{m}}}{A_{\mathrm{m}}}\left(A_{\mathrm{m}}\right.$ is the true projected contact area measured at the maximum load $P_{\mathrm{m}}$ ), can be identified with the hardness of the indented material. The maximum indentation depth $h_{\mathrm{m}}$ occurs at $P_{\mathrm{m}}$, and the initial unloading slope is defined as $\left.\frac{\mathrm{d} P_{\mathrm{u}}}{\mathrm{d} h}\right|_{h_{\mathrm{m}}}$, where $P_{\mathrm{u}}$ is the unloading force. The $W_{\mathrm{t}}$ term is the total work done by load $P$ during loading, $W_{\mathrm{e}}$ is the released (elastic) work during unloading, and the stored (plastic) work $W_{\mathrm{p}}=W_{\mathrm{t}}-W_{\mathrm{e}}$. The residual indentation depth after complete unloading is $h_{\mathrm{r}}$.

As discussed by Giannakopoulos and Suresh [20], $C,\left.\frac{\mathrm{d} P_{\mathrm{u}}}{\mathrm{d} h}\right|_{h_{\mathrm{m}}}$ and $\frac{h_{\mathrm{r}}}{h_{\mathrm{m}}}$ are three independent quantities that can be directly obtained from a single $P-h$ curve.

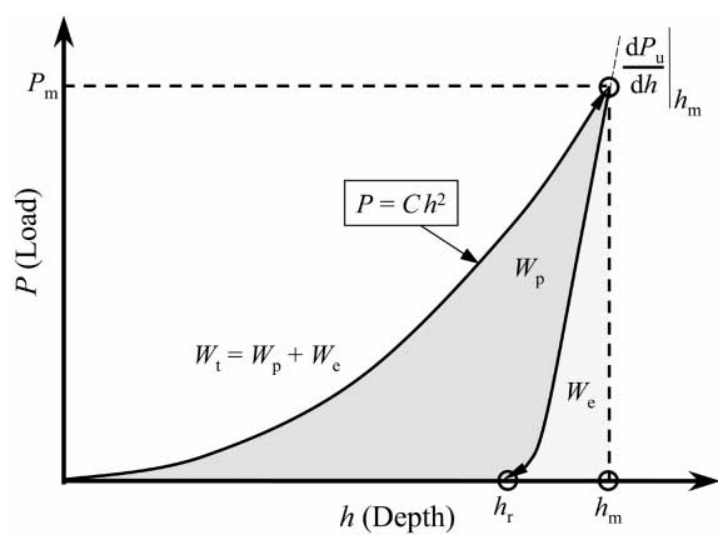

Fig. 1. Schematic illustration of a typical $P-h$ response of an elasto-plastic material to instrumented sharp indentation. 
The question remains whether these parameters are sufficient to uniquely determine the indented material's elasto-plastic properties.

Plastic behavior of many pure and alloyed engineering metals can be closely approximated by a power law description, as shown schematically in Fig. 2. A simple elasto-plastic, true stress-true strain behavior is assumed to be

$$
\sigma= \begin{cases}E \varepsilon, & \text { for } \sigma \leq \sigma_{y} \\ R \varepsilon^{n}, & \text { for } \sigma \geq \sigma_{y}\end{cases}
$$

where $E$ is the Young's modulus, $R$ a strength coefficient, $n$ the strain hardening exponent, $\sigma_{\mathrm{y}}$ the initial yield stress and $\varepsilon_{\mathrm{y}}$ the corresponding yield strain, such that

$$
\sigma_{\mathrm{y}}=E \varepsilon_{\mathrm{y}}=R \varepsilon_{\mathrm{y}}^{n}
$$

Here the yield stress $\sigma_{\mathrm{y}}$ is defined at zero offset strain. The total effective strain, $\varepsilon$, consists of two parts, $\varepsilon_{\mathrm{y}}$ and $\varepsilon_{\mathrm{p}}$ :

$$
\varepsilon=\varepsilon_{\mathrm{y}}+\varepsilon_{\mathrm{p}}
$$

where $\varepsilon_{\mathrm{p}}$ is the nonlinear part of the total effective strain accumulated beyond $\varepsilon_{\mathrm{y}}$. With equations (3) and (4), when $\sigma>\sigma_{\mathrm{y}}$, equation (2) becomes

$$
\sigma=\sigma_{\mathrm{y}}\left(1+\frac{E}{\sigma_{\mathrm{y}}} \varepsilon_{\mathrm{p}}\right)^{n}
$$

To complete the material constitutive description,

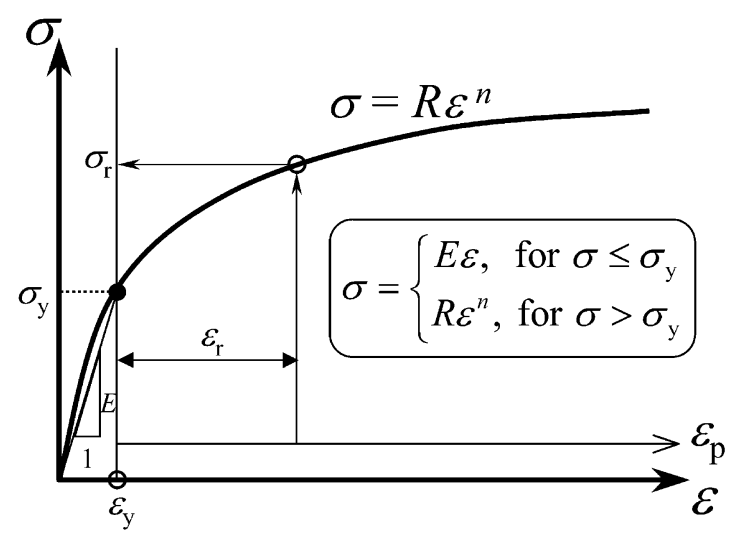

Fig. 2. The power law elasto-plastic stress-strain behavior used in the current study.
Poisson's ratio is designated as $v$, and the incremental theory of plasticity with von Mises effective stress $\left(J_{2}\right.$ flow theory) is assumed.

With the above assumptions and definitions, a material's elasto-plastic behavior is fully determined by the parameters $E, v, \sigma_{\mathrm{y}}$ and $n$. Alternatively, with the constitutive law defined in equation (2), the power law strain hardening assumption reduces the mathematical description of plastic properties to two independent parameters. This pair could be described as a representative stress $\sigma_{\mathrm{r}}$ (defined at $\varepsilon_{\mathrm{p}}=\varepsilon_{\mathrm{r}}$, where $\varepsilon_{\mathrm{r}}$ is a representative strain) and the strain-hardening exponent $n$, or as $\sigma_{\mathrm{y}}$ and $\sigma_{\mathrm{r}}$.

\subsection{Dimensional analysis and universal functions}

Cheng and Cheng [11, 12] and Tunvisut et al. [25] have used dimensional analysis to propose a number of dimensionless universal functions, with the aid of computational data points calculated via the Finite Element Method (FEM). Here, a number of new dimensionless functions are described in the following paragraphs.

As discussed in Section 2.1, one can use a material parameter set $\left(E, v, \sigma_{\mathrm{y}}\right.$ and $\left.n\right),\left(E, v, \sigma_{\mathrm{r}}\right.$ and $\left.n\right)$ or $(E$, $v, \sigma_{\mathrm{y}}$ and $\sigma_{\mathrm{r}}$ ) to describe the constitutive behavior. Therefore, the specific functional forms of the universal dimensionless functions are not unique (but different definitions are interdependent if power law strain hardening is assumed). For instrumented sharp indentation, a particular material constitutive description (e.g., power-law strain hardening) yields its own distinct set of dimensionless functions. One may choose to use any plastic strain to be the representative strain $\varepsilon_{\mathrm{r}}$, where the corresponding $\sigma_{\mathrm{r}}$ is used to describe the dimensionless functions. However, the representative strain which best normalizes a particular dimensionless function with respect to strain hardening will be a distinct value.

Here, we present a set of universal dimensionless functions and their closed-form relationship between indentation data and elasto-plastic properties (within the context of the present computational results). This set of functions leads to new algorithms for accurately predicting the $P-h$ response from known elasto-plastic properties (forward algorithms) and new algorithms for systematically extracting the indented material's elasto-plastic properties from a single set of $P-h$ data (reverse algorithms).

For a sharp indenter (conical, Berkovich or Vickers, with fixed indenter shape and tip angle) indenting normally into a power law elasto-plastic solid, the load $P$ can be written as

$$
P=P\left(h, E, v, E_{\mathrm{i}}, v_{\mathrm{i}}, \sigma_{\mathrm{y}}, n\right),
$$

where $E_{\mathrm{i}}$ is Young's modulus of the indenter, and $v_{\mathrm{i}}$ is its Poisson's ratio. This functionality is often simplified (e.g., [29]) by combining elasticity effects of an elastic indenter and an elasto-plastic solid as 


$$
P=P\left(h, E^{*}, \sigma_{\mathrm{y}}, n\right),
$$

(7) $1 \mu \mathrm{m}$ ), then the scale dependent effects become small and may be ignored. In the current study, any scale dependent effects are assumed to be insignificant. It is clear from equations (11) and (12) that, for an isotropic and homogeneous material, $P=C h^{2}$ is the natural outcome of the dimensional analysis for a

$$
E^{*}=\left[\frac{1-v^{2}}{E}+\frac{1-v_{\mathrm{i}}^{2}}{E_{\mathrm{i}}}\right]^{-1}
$$

Alternatively, equation (7) can be written as

$$
P=P\left(h, E^{*}, \sigma_{\mathrm{r}}, n\right)
$$

or

$$
P=P\left(h, E^{*}, \sigma_{\mathrm{y}}, \sigma_{\mathrm{r}}\right)
$$

Applying the $\Pi$ theorem in dimensional analysis, equation (9) becomes

$$
P=\sigma_{\mathrm{r}} h^{2} \Pi_{1}\left(\frac{E^{*}}{\sigma_{\mathrm{r}}}, n\right),
$$

and thus

$$
C=\frac{P}{h^{2}}=\sigma_{\mathrm{r}} \Pi_{1}\left(\frac{E^{*}}{\sigma_{\mathrm{r}}}, n\right) .
$$

where $\Pi_{1}$ is a dimensionless function. Similarly, applying the $\Pi$ theorem to equation (10), loading curvature $C$ may alternatively be expressed as

$$
C=\frac{P}{h^{2}}=\sigma_{\mathrm{y}} \Pi_{1}^{\mathrm{A}}\left(\frac{E^{*}}{\sigma_{\mathrm{y}}}, \frac{\sigma_{\mathrm{r}}}{\sigma_{\mathrm{y}}}\right)
$$

or

$$
C=\frac{P}{h^{2}}=\sigma_{\mathrm{r}} \Pi_{1}^{\mathrm{B}}\left(\frac{E^{*}}{\sigma_{\mathrm{r}}}, \frac{\sigma_{\mathrm{y}}}{\sigma_{\mathrm{r}}}\right)
$$

where $\Pi_{1}^{\mathrm{A}}$ and $\Pi_{1}^{\mathrm{B}}$ are dimensionless functions. The dimensionless functions given in equations (11) and (12) are different from those proposed in [11, 12], where the normalization was taken with respect to $E^{*}$ instead of $\sigma_{\mathrm{r}}$ or $\sigma_{\mathrm{y}}$.

During nanoindentation experiments, especially when the indentation depth is about $100-1000 \mathrm{~nm}$, size-scale-dependent indentation effects have been postulated (e.g., $[8,30,31])$. These possible sizescale-dependent effects on hardness have been modeled using higher order theories (e.g., [30, 31]). If the indentation is sufficiently deep (typically deeper than sharp indenter; loading curvature $C$ is a material constant which is independent of indentation depth. It is also noted that, depending on the choices of $\left(\varepsilon_{\mathrm{r}}, \sigma_{\mathrm{r}}\right)$, there are an infinite number of ways to define the dimensionless function $\Pi_{1}$. However, with the assumption of power-law strain hardening, it can be shown that one definition of $\Pi_{1}$ is easily converted to another definition.

If the unloading force is represented as $P_{\mathrm{u}}$, the unloading slope is given by

$$
\frac{\mathrm{d} P_{\mathrm{u}}}{\mathrm{d} h}=\frac{\mathrm{d} P_{\mathrm{u}}}{\mathrm{d} h}\left(h, h_{\mathrm{m}}, E, v, E_{\mathrm{i}}, v_{\mathrm{i}}, \sigma_{\mathrm{r}}, h\right)
$$

or, assuming that elasticity effects are characterized by $E^{*}$, the unloading slope is given by

$$
\frac{\mathrm{d} P_{\mathrm{u}}}{\mathrm{d} h}=\frac{\mathrm{d} P_{\mathrm{u}}}{\mathrm{d} h}\left(h, h_{\mathrm{m}}, E^{*}, \sigma_{\mathrm{r}}, n\right)
$$

Dimensional analysis yields

$$
\frac{\mathrm{d} P_{\mathrm{u}}}{\mathrm{d} h}=E^{*} h \Pi_{2}^{0}\left(\frac{h_{\mathrm{m}}}{h}, \frac{\sigma_{\mathrm{r}}}{E^{*}}, n\right)
$$

Evaluating equation (14) at $h=h_{\mathrm{m}}$ gives

$$
\begin{gathered}
\left.\frac{\mathrm{d} P_{\mathrm{u}}}{\mathrm{d} h}\right|_{h=h_{\mathrm{m}}}=E^{*} h_{\mathrm{m}} \Pi_{2}^{0}\left(1, \frac{\sigma_{\mathrm{r}}}{E^{*}}, n\right) \\
=E^{*} h_{\mathrm{m}} \Pi_{2}\left(\frac{E^{*}}{\sigma_{\mathrm{r}}}, n\right)
\end{gathered}
$$

Similarly, $P_{\mathrm{u}}$ itself can be expressed as

$$
P_{\mathrm{u}}=P_{\mathrm{u}}\left(h, h_{\mathrm{m}}, E^{*}, \sigma_{\mathrm{r}}, n\right)=E^{*} h^{2} \Pi_{\mathrm{u}}\left(\frac{h_{\mathrm{m}}}{h}, \frac{\sigma_{\mathrm{r}}}{E^{*}}, n\right)
$$

When $P_{\mathrm{u}}=0$, the specimen is fully unloaded and, thus, $h=h_{\mathrm{r}}$. Therefore, upon complete unloading,

$$
0=\Pi_{\mathrm{u}}\left(\frac{h_{\mathrm{m}}}{h_{\mathrm{r}}}, \frac{\sigma_{\mathrm{r}}}{E^{*}}, n\right)
$$

Rearranging equation (17), 


$$
\frac{h_{\mathrm{r}}}{h_{\mathrm{m}}}=\Pi_{3}\left(\frac{\sigma_{\mathrm{r}}}{E^{*}}, n\right)
$$

Thus, the three universal dimensionless functions, $\Pi_{1}$, $\Pi_{2}$ and $\Pi_{3}$, can be used to relate the indentation response to mechanical properties.

\subsection{Computational model}

Axisymmetric two-dimensional and full threedimensional finite element models were constructed to simulate the indentation response of elasto-plastic solids. Figure 3(a) schematically shows the conical indenter, where $\theta$ is the included half angle of the indenter, $h_{\mathrm{m}}$ is the maximum indentation depth, and $a_{\mathrm{m}}$ is the contact radius measured at $h_{\mathrm{m}}$. The true projected contact area $A_{\mathrm{m}}$, with pile-up or sink-in effects taken into account, for a conical indenter is thus

$$
A_{\mathrm{m}}=\pi a_{\mathrm{m}}^{2}
$$

Figure 3(b) shows the mesh design for axisymmetric calculations. The semi-infinite substrate of the indented solid was modeled using 8100 four-noded, bilinear axisymmetric quadrilateral elements, where a fine mesh near the contact region and a gradually coarser mesh further from the contact region were designed to ensure numerical accuracy. At the maximum load, the minimum number of contact elements in the contact zone was no less than 16 in each FEM computation. The mesh was well-tested for convergence and was determined to be insensitive to far-field boundary conditions.

Three-dimensional finite element models incorporating the inherent six-fold or eight-fold symmetry of a Berkovich or a Vickers indenter, respectively, were also constructed. A total of 11,150 and 10,401 eightnoded, isoparametric elements was used for Berkovich and Vickers indentation, respectively. Figure 3(c) shows the overall mesh design for the Berkovich indentation, while Fig. 3(d) details the area that directly contacts the indenter tip. Computations were performed using the general purpose finite element package ABAQUS [32]. The three-dimensional mesh design was verified against the three-dimensional results obtained from the mesh used previously by Larsson et al. [27]. Unless specified otherwise, large deformation theory was assumed throughout the analysis.

For a conical indenter, the projected contact area is $A=\pi h^{2} \tan ^{2} \theta$; for a Berkovich indenter, $A=24.56 h^{2}$; for a Vickers indenter, $A=24.50 h^{2}$. In this study, the three-dimensional indentation induced via Berkovich or Vickers geometries was approximated with axisymmetric two-dimensional models by choosing the apex angle $\theta$ such that the projected area/depth of the two-dimensional cone was the same as that for the Berkovich or Vickers indenter. For both Berkovich and Vickers indenters, the corre- (a)

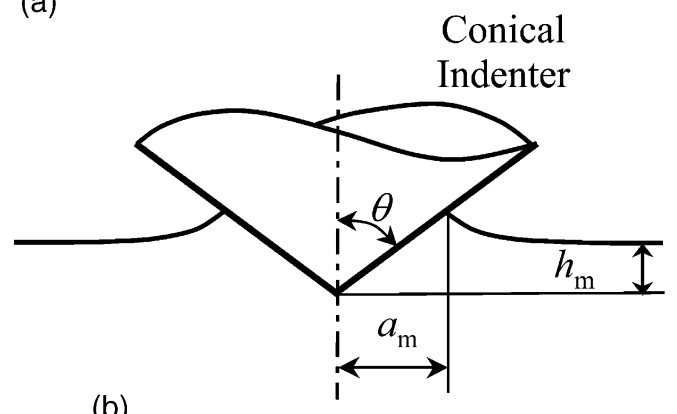

(b)

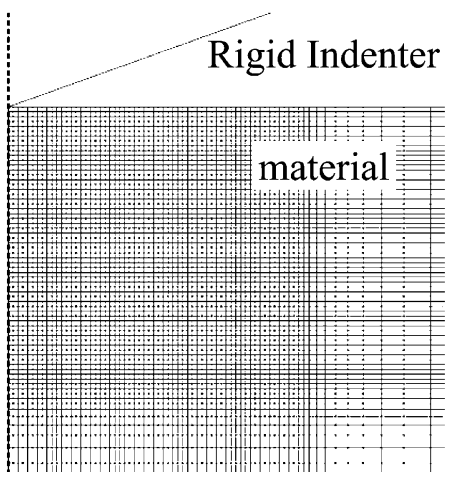

(c)

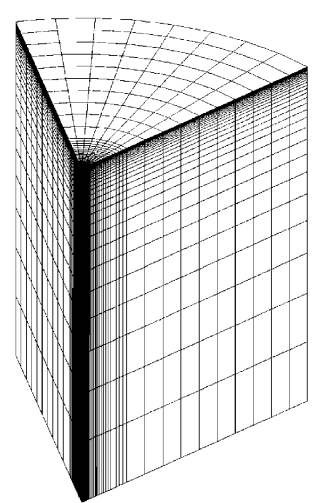

(d)

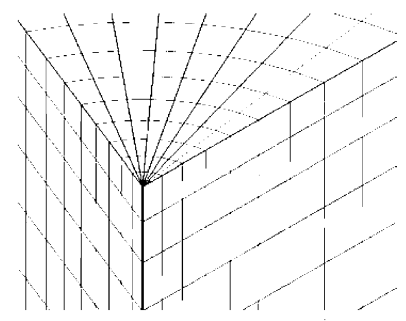

Fig. 3. Computational modeling of instrumented sharp indentation. (a) Schematic drawing of the conical indenter, (b) mesh design for axisymmetric finite element calculations, (c) overall mesh design for the Berkovich indentation calculations, and (d) detailed illustration of the area that directly contacts the indenter tip in (c). 
sponding apex angle $\theta$ of the equivalent cone was chosen as $70.3^{\circ}$. Axisymmetric two-dimensional computational results will be referenced in the remainder of the paper unless otherwise specified. In all finite element computations, the indenter was modeled as a rigid body, and the contact was modeled as frictionless. Detailed pile-up and sink-in effects were more accurately accounted for by the large deformation FEM computations, as compared to small deformation computations.

\subsection{Comparison of experimental and computational results}

Two aluminum alloys were obtained for experimental investigation: 6061-T6511 and 7075-T651 aluminum, both in the form of $2.54 \mathrm{~cm}$ diameter, extruded round bar stock. Two compression specimens $(0.5 \mathrm{~cm}$ diameter, $0.75 \mathrm{~cm}$ height $)$ were machined from each bar such that the compression axis was parallel to the extrusion direction. Simple uniaxial compression tests were conducted on a servo-hydraulic universal testing machine at a crosshead speed of $0.2 \mathrm{~mm} / \mathrm{min}$. Crosshead displacement was obtained from a calibrated LVDT (linear voltagedisplacement transducer). As each specimen was compressed to $45 \%$ engineering strain, the specimen ends were lubricated with Teflon ${ }^{\mathrm{TM}}$ lubricant to prevent barreling. Intermittent unloading was conducted to allow for repeated measurement of Young's modulus and relubrication of the specimen ends. Recorded load-displacement data were converted to true stresstrue strain data. Although the true stress-true strain responses were well approximated by power law fits, these experimental stress-strain data were used as direct input for FEM simulations, rather than the mathematical approximations (see Fig. 4). For 7075T651 aluminum, the measured Young's modulus was $E=70.1 \mathrm{GPa}$; and for 6061-T6511 aluminum, $E=66.8 \mathrm{GPa}$.

Indentation specimens were machined from the same round bar stock as discs of the bar diameter (3 $\mathrm{mm}$ thickness). Each specimen was polished to 0.06 $\mu \mathrm{m}$ surface finish with colloidal silica. These speci-

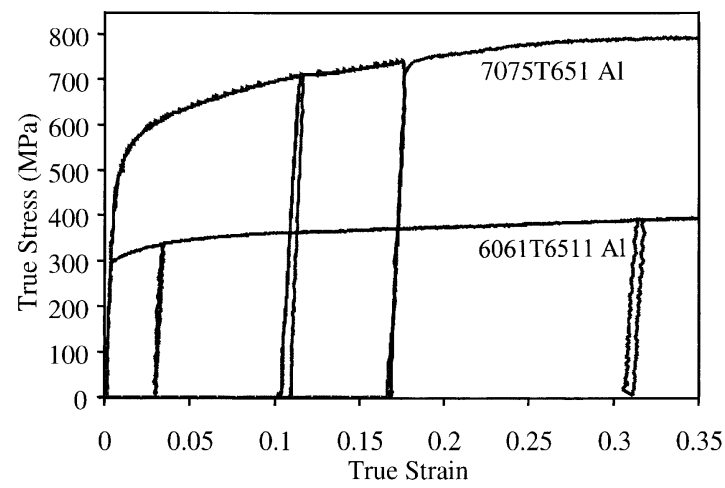

Fig. 4. Experimental uniaxial compression stress-strain curves of both 6061-T6511 aluminum and 7075-T651 aluminum specimens, respectively.

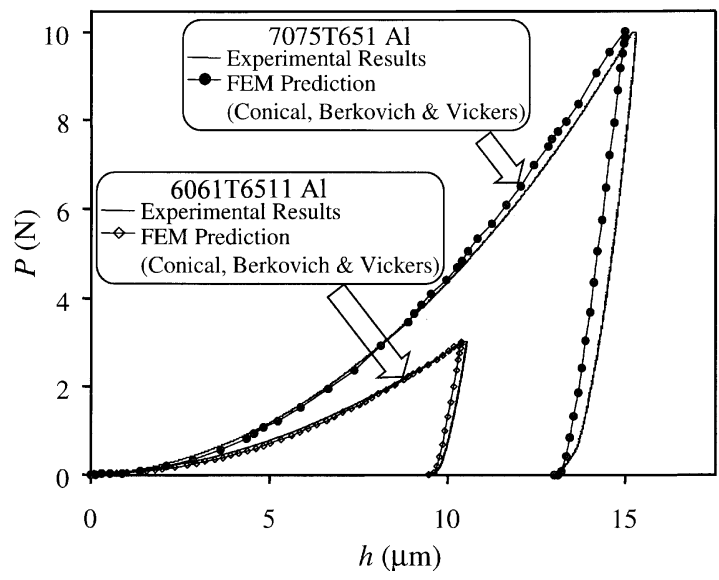

Fig. 5. Experimental versus computational indentation responses of both the 7075-T651 aluminum and 6061-T6511 aluminum specimens, respectively.

mens were then indented on a commercial nanoindenter (MicroMaterials, Wrexham, UK) with a Berkovich diamond indenter at a loading/unloading rate of approximately $0.2 \mathrm{~N} / \mathrm{min}$. For each of three maximum loads $(3,10$, and $20 \mathrm{~N})$, five tests were conducted on two consecutive days, for a total of ten tests per load in each specimen. Figure 5 shows the typical indentation responses of both the 7075-T651 aluminum and 6061-T6511 aluminum specimens, respectively. The corresponding finite element computations using conical, Berkovich and Vickers indenters are also plotted in Fig. 5. Figure 6 shows the equivalent plastic strain (PEEQ) within the 7075-T651 aluminum near the tip of the conical indenter, indicating that the majority of the volume directly beneath the indenter experienced strains exceeding $15 \%$. Assuming only the $\sigma-\varepsilon$ constitutive response obtained from experimental uniaxial compression, the computational $P-$ $h$ curves agree well with the experimental curves, as shown in Fig. 5. The computational $P-h$ responses of the conical, Berkovich and Vickers indentations were found to be virtually identical.

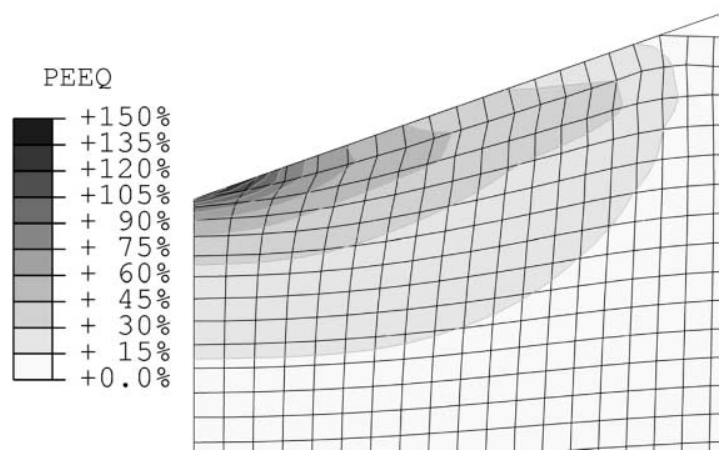

Fig. 6. Contour plot of the equivalent plastic strain (PEEQ) within the 7075-T651 aluminum near the tip of the conical indenter, indicating that the majority of the volume directly beneath the indenter experienced strains exceeding $15 \%$. 
Table 1. Four cases studied to compare large vs small deformation theory

\begin{tabular}{llllll}
\hline Case & System & $E(\mathrm{GPa})$ & Yield strength $(\mathrm{MPa})$ & $n$ & $v$ \\
\hline A & Zinc alloy & 9 & 300 & 0.05 & 0.3 \\
B & Refractory alloy & 80 & 1500 & 0.05 & 0.3 \\
C & Aluminum alloy & 70 & 300 & 0.05 & 0.28 \\
D & Steel & 210 & 500 & 0.1 & 0.27 \\
\hline
\end{tabular}

\subsection{Large deformation vs small deformation}

Giannakopoulos et al. [18], Larsson et al. [27], Giannakopoulos and Suresh [20], and Venkatesh et al. [21] have proposed a systematic methodology to extract elasto-plastic properties from a single $P-h$ curve. The loading curvature $C$ was given as

$$
C=M_{1} \sigma_{0.29}\left(1+\frac{\sigma_{y}}{\sigma_{0.29}}\right)\left[M_{2}+\ln \left(\frac{E^{*}}{\sigma_{y}}\right)\right]
$$

where $M_{1}$ and $M_{2}$ are computationally derived constants which depend on indenter geometry. It is interesting to note that, after rewriting $\sigma_{0.29}\left(1+\sigma_{\mathrm{y}} / \sigma_{0.29}\right)$ as $\sigma_{y}\left(1+\sigma_{0.22} / \sigma_{y}\right)$, equation (20) is consistent with equation (12a).

Figure 7 shows the comparison between the large deformation solution, small deformation solution and the predictions from equation (20), using the four model materials listed in Venkatesh et al. [21] (see Table 1). From Fig. 7, it is evident that equation (20) agrees well with the small deformation results and that, for all four cases studied, large deformation theory always predicts a stiffer loading response.

In addition, 76 different cases covering material parameters of most engineering metals were studied computationally. Detailed examination showed that large deformation solutions are not readily described by equation (20), but rather are better approximated within $\pm 10 \%$ (for the conical indenter with $\theta=70.3^{\circ}$ ) by a new universal function given by

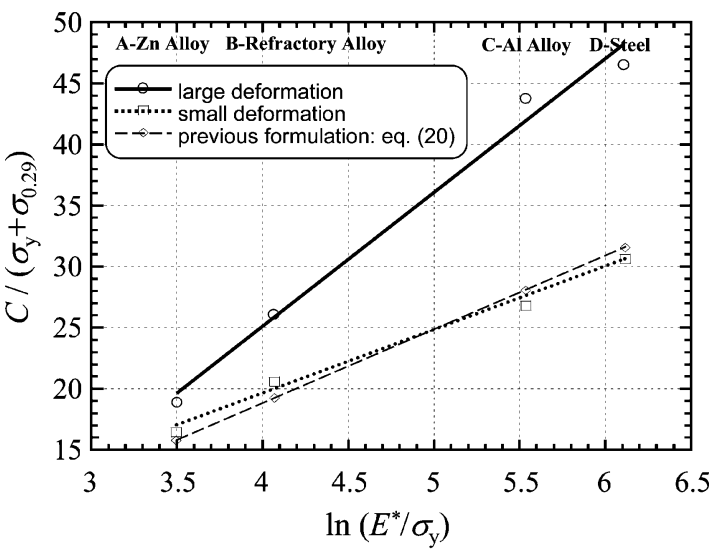

Fig. 7. Comparison between the large deformation solution, small deformation solution and the previous formulation [equation (20)] using four model materials. For all four cases studied, large deformation theory always predicts a stiffer loading response.

$$
C=N_{1} \sigma_{0.29}\left(1+\frac{\sigma_{\mathrm{y}}}{\sigma_{0.29}}\right)\left[N_{2}+\ln \left(\frac{E^{*}}{\sigma_{0.29}}\right)\right]
$$

where $N_{1}=9.4509$ and $N_{2}=-1.2433$ are computationally derived constants specific to the indenter geometry. This expression is consistent with the dimensionless function shown in equation (12b).

\section{COMPUTATIONAL RESULTS}

A comprehensive parametric study of 76 additional cases was conducted (see Appendix A for a complete list of parameters). These cases represented the range of parameters of mechanical behavior found in common engineering metals: that is, Young's modulus $E$ ranged from 10 to $210 \mathrm{GPa}$, yield strength $\sigma_{\mathrm{y}}$ from 30 to $3000 \mathrm{MPa}$, strain hardening exponent $n$ from 0 to 0.5 , and Poisson's ratio $v$ was fixed at 0.3 . The axisymmetric finite element model was used to obtain computational results unless otherwise specified.

\subsection{Representative strain and universal dimen- sionless functions}

The first dimensionless function of interest is $\Pi_{1}$ in equation (11). From equation (11),

$$
\Pi_{1}\left(\frac{E^{*}}{\sigma_{\mathrm{r}}}, n\right)=\frac{C}{\sigma_{\mathrm{r}}}
$$

The specific functional form of $\Pi_{1}$ depends on the choice of $\varepsilon_{\mathrm{r}}$ and $\sigma_{\mathrm{r}}$. Figure 8 shows the computationally obtained results using three different values of $\varepsilon_{\mathrm{r}}$ (i.e., $\varepsilon_{\mathrm{p}}=0.01,0.033$ and 0.29 ) and the corresponding $\sigma_{\mathrm{r}}$. The results in Fig. 8 indicate that for $\varepsilon_{\mathrm{r}}<0.033, \Pi_{1}$ increased with increasing $n$; for $\varepsilon_{\mathrm{r}}>$ $0.033, \Pi_{1}$ decreased with increasing $n$. Minimizing the relative errors using a least squares algorithm, it is confirmed that when $\varepsilon_{\mathrm{r}}=0.033$, a polynomial function $\Pi_{1}\left(\frac{E^{*}}{\sigma_{0.033}}\right)={\frac{C}{\sigma_{0.033}} \dagger}^{\dagger}$ fits all 76 data points within $\mathrm{a} \pm 2.85 \%$ error [see Fig. 8(b)]. A representative strain of $\varepsilon_{\mathrm{r}}=0.033$ was thus identified. The corresponding dimensionless function $\Pi_{1}$ normalized with respect to $\sigma_{0.033}$ was found to be independent of the strain hardening exponent $n$. This result indicates that, for a given value of $E^{*}$, all power law plastic, true stresstrue strain responses that exhibit the same true stress

$\dagger$ See Appendix B for a complete listing of the function. 

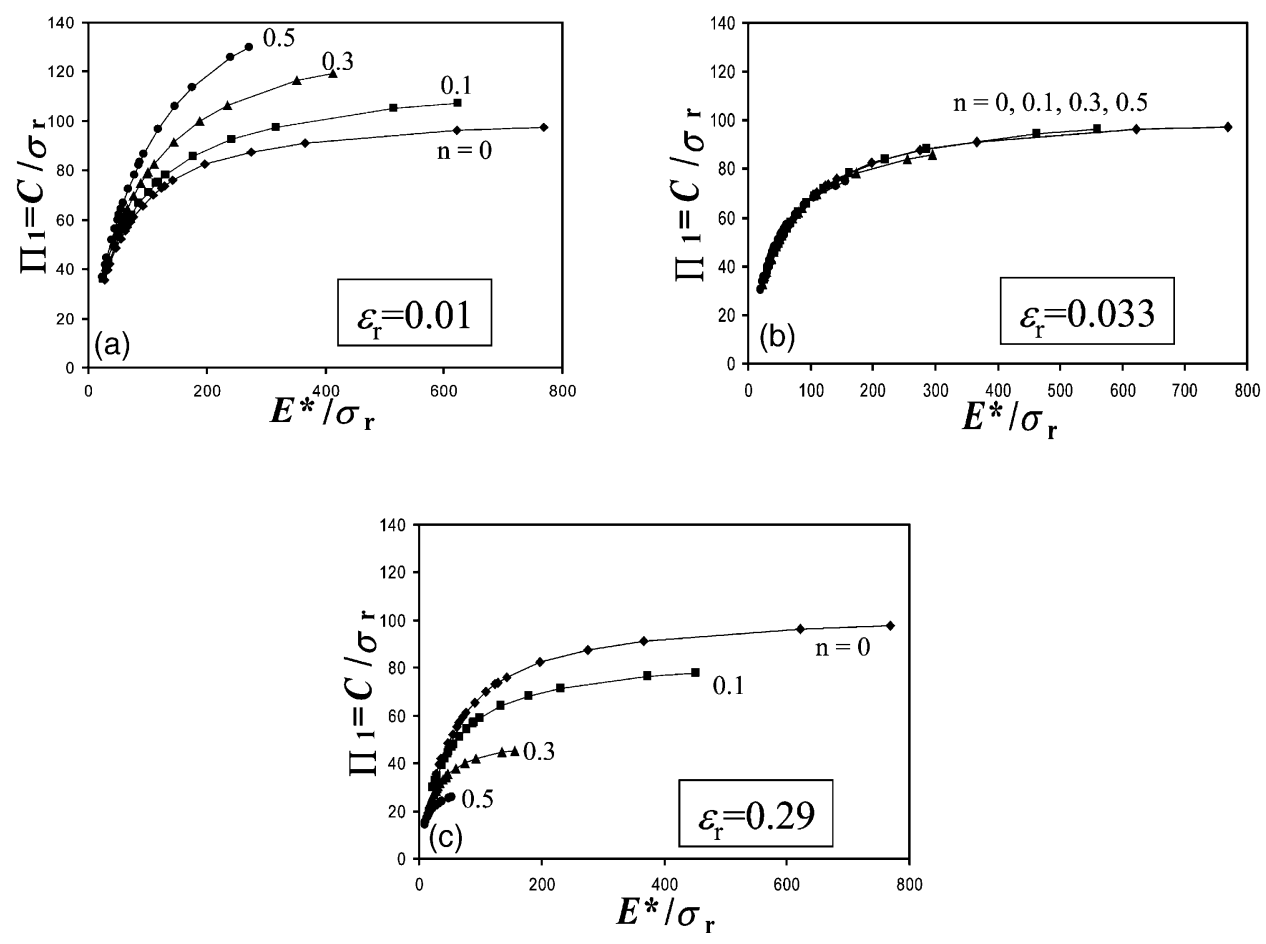

Fig. 8. Dimensionless function $\Pi_{1}$ constructed using three different values of $\varepsilon_{\mathrm{r}}\left(i . e ., \varepsilon_{\mathrm{p}}=0.01,0.033\right.$ and 0.29$)$ and the corresponding $\sigma_{\mathrm{r}}$, respectively. For $\varepsilon_{\mathrm{r}}<0.033, \Pi_{1}$ increased with increasing $n$; for $\varepsilon_{\mathrm{r}}>0.033, \Pi_{1}$ decreased with increasing $n$. A representative plastic strain $\varepsilon_{\mathrm{r}}=0.033$ can be identified as a strain level which allows for the construction of $\Pi_{1}$ to be independent of strain hardening exponent $n$.

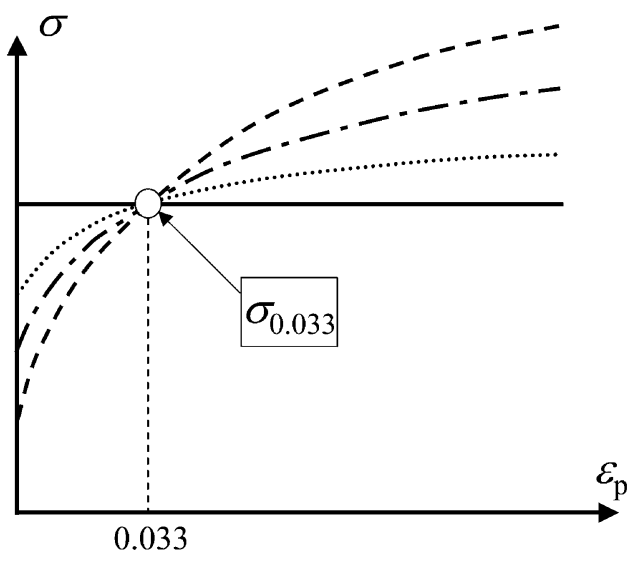

Fig. 9. For a given value of $E^{*}$, all power law plastic, true stress-true strain responses that exhibit the same true stress at $3.3 \%$ true plastic strain give the same indentation loading curvature $C$. A collection of such plastic stress-strain curves are schematically illustrated in the figure.

at $3.3 \%$ true plastic strain give the same indentation loading curvature $C$ (see Fig. 9). It is noted that this result was obtained within the specified range of material parameters using the material constitutive behavior defined by equation (2).

Figure 10 show the dimensionless functions $\Pi_{2}$ and $\Pi_{3}$. Within a $\pm 2.5 \%$ and a $\pm 0.77 \%$ error,
$\Pi_{2}\left(\frac{E^{*}}{\sigma_{\mathrm{r}}}, n\right)=\left.\frac{1 \mathrm{~d} P_{\mathrm{u}}}{E^{*} h_{\mathrm{m}} \mathrm{d} h}\right|_{h_{\mathrm{m}}} ^{\dagger}$ and $\Pi_{3}\left(\frac{\sigma_{\mathrm{r}}}{E^{*}}, n\right)={\frac{h_{\mathrm{r}}}{h_{\mathrm{m}}}}^{\dagger}$ fit all 76 sets of computed data shown in Fig. 10(a) and (b), respectively.

Several other (approximate) dimensionless functions were also computationally derived. Figure 11(a) shows the dimensionless function $\Pi_{4}\left(\frac{h_{\mathrm{r}}}{h_{\mathrm{m}}}\right)=\frac{p_{\mathrm{ave}^{\dagger}}^{\dagger}}{E^{*}}$ within $\pm 13.85 \%$ of the computationally obtained values for the 76 cases studied. It is noted that the verified range for $\Pi_{4}$ is $0.5<\frac{h_{\mathrm{r}}}{h_{\mathrm{m}}}<0.98$. Figure 11(b) shows dimensionless function $\Pi_{5}\left(\frac{h_{\mathrm{r}}}{h_{\mathrm{m}}}\right)={\frac{W_{\mathrm{p}}^{\dagger}}{W_{\mathrm{t}}}}^{\dagger}$ within $\pm 2.38 \%$ of the numerically computed values for the 76 cases. The verified range for function $\Pi_{5}$ is the same as that for $\Pi_{4}$, i.e. $0.5<\frac{h_{\mathrm{r}}}{h_{\mathrm{m}}}<0.98$. From Fig. $11(\mathrm{~b})$, it is obvious that $\frac{W_{\mathrm{p}}}{W_{\mathrm{t}}}=\frac{h_{\mathrm{r}}}{h_{\mathrm{m}}}$ is not a good approximation except when $\frac{h_{\mathrm{r}}}{h_{\mathrm{m}}}$ approaches unity.

According to King [33],

$\dagger$ See Appendix B for a complete listing of the function. 
(a)

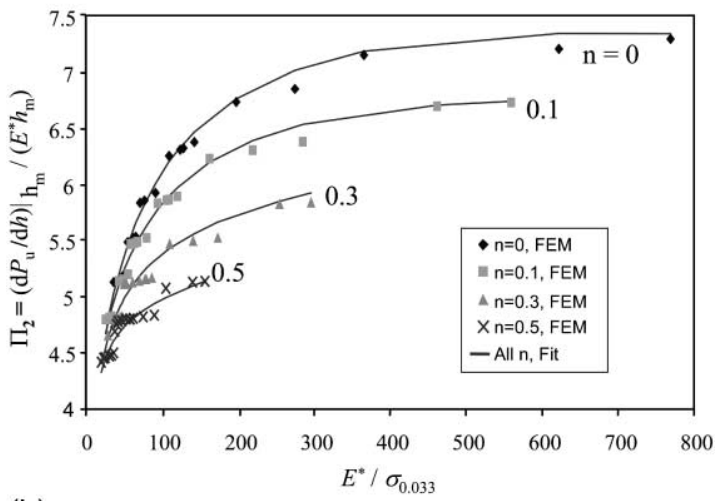

(b)

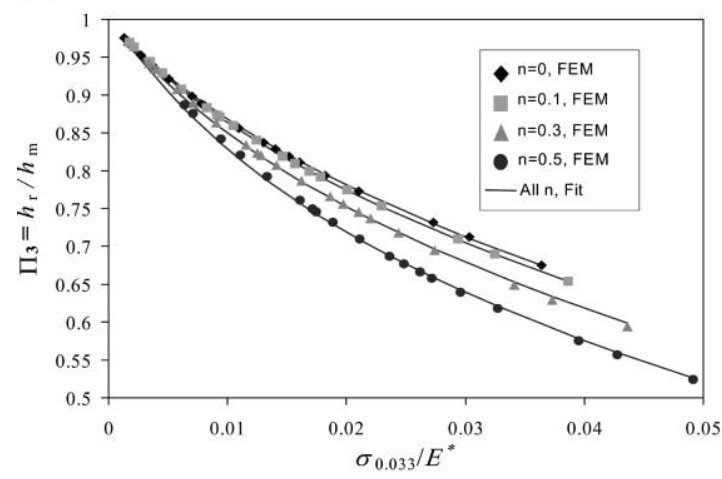

Fig. 10. Dimensionless functions (a) $\Pi_{2}\left(\frac{E^{*}}{\sigma_{\mathrm{r}}}, n\right)$ and (b)

$$
\begin{gathered}
\Pi_{3}\left(\frac{\sigma_{\mathrm{r}}}{E^{*}}, n\right) . \\
E^{*}=\left.\frac{1 \mathrm{~d} P_{\mathrm{u}}}{c^{*} \sqrt{A_{\mathrm{m}}} \mathrm{d} h}\right|_{h_{\mathrm{m}}}
\end{gathered}
$$

where linear elastic analysis gives $c^{*}=1.167$ for the Berkovich indenter, 1.142 for the Vickers indenter and 1.128 for the conical indenter. Large deformation elasto-plastic analysis of the 76 cases showed that $c^{*} \approx 1.1957$ (within $\pm 0.9 \%$ error) for the conical indenter with $\theta=70.3^{\circ}$. This value of $c^{*}$, which takes into account the elasto-plastic finite deformation prior to the unloading, is about $6 \%$ higher than the smalldeformation, linear-elastic solution. It is noted that the initial unloading response is expected to be entirely elastic, and the linear elastic solution is quite accurate compared to the large deformation solution. Assuming the same relative influence of the large deformation elasto-plastic solution on the elastic solution for the Berkovich and Vickers geometries, the adjusted values of $c^{*}$ were proposed to be 1.2370 and 1.2105 , respectively. This completes another important dimensionless function $\Pi_{6}$,

$$
\Pi_{6}=\left.\frac{1}{E^{*} \sqrt{A_{\mathrm{m}}}} \frac{\mathrm{d} P_{\mathrm{u}}}{\mathrm{d} h}\right|_{h_{\mathrm{m}}}=c^{*}
$$

(a)

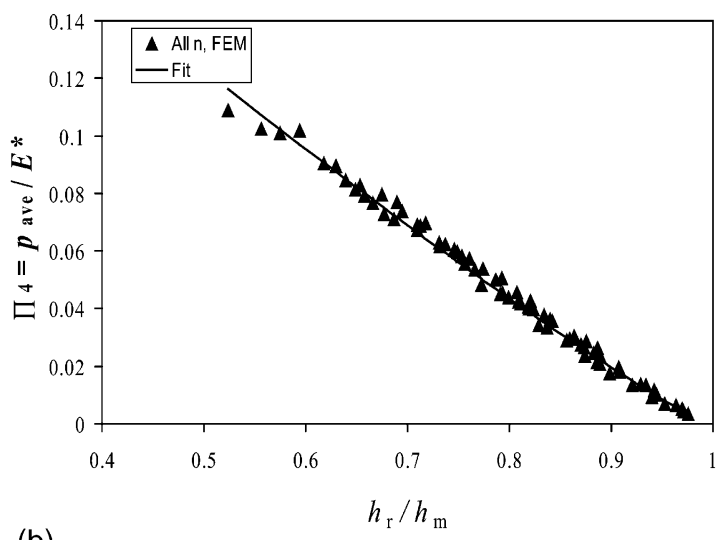

(b)

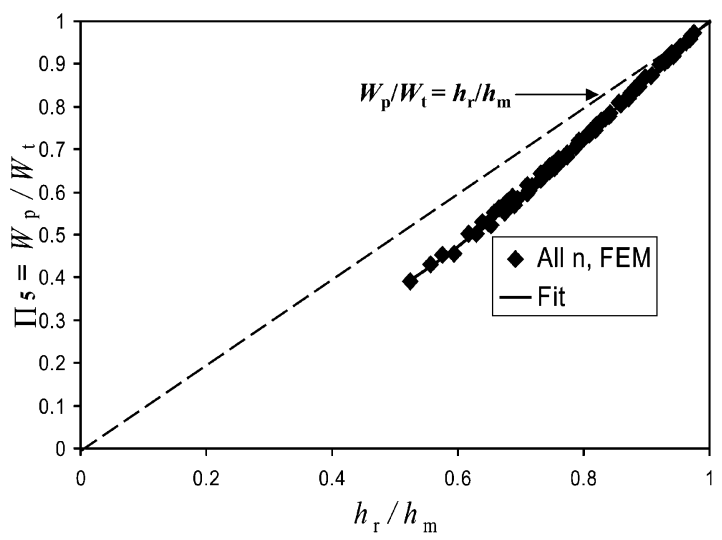

Fig. 11. Dimensionless functions (a) $\Pi_{4}\left(\frac{h_{\mathrm{r}}}{h_{\mathrm{m}}}\right)$ and (b) $\Pi_{5}\left(\frac{h_{\mathrm{r}}}{h_{\mathrm{m}}}\right)$.

For a conical indenter with $\theta=70.3^{\circ}$, noting that $A_{\mathrm{m}}=\pi a_{\mathrm{m}}^{2}$, equation (24) can be rewritten as

$$
\Pi_{6 \mathrm{C}}=\left.\frac{1 \mathrm{~d} P_{\mathrm{u}}}{E^{*} a_{\mathrm{m}} \mathrm{d} h}\right|_{h_{\mathrm{m}}}=c^{*} \sqrt{\pi} \approx 2.12
$$

Note that equation (25) is simply a revision of equation (23) in light of the computationally derived values of $c^{*}$. In Oliver and Pharr [5], $c^{*} \sqrt{\pi}=2$ (i.e., $c^{*}=1.128$, the linear elastic solution) was used. Table 2 tabulates the values of $c^{*}$ used in the current study and in the literature.

Table 2. The values of $c^{*}$ used in the current study

\begin{tabular}{lll}
\hline$c^{*}$ & $\begin{array}{l}\text { Small deformation } \\
\text { linear elastic } \\
\text { solution }^{\mathrm{a}}\end{array}$ & $\begin{array}{l}\text { Large deformation } \\
\text { elasto-plastic } \\
\text { solution }^{\mathrm{b}}\end{array}$ \\
\hline Conical & 1.128 & 1.1957 \\
Berkovich & 1.167 & 1.2370 \\
Vickers & 1.142 & 1.2105 \\
\hline
\end{tabular}

${ }^{\text {a }}$ King [33]

b Proposed in the current study 
It is noted that $\Pi_{3}$ and $\Pi_{4}$ are interdependent, i.e., function $\Pi_{4}$ together with dimensionless functions $\Pi_{1}, \Pi_{2}$ and $\Pi_{6}$, can be used to solve for $\Pi_{3}$. Function $\Pi_{5}$ relates $\frac{W_{\mathrm{p}}}{W_{\mathrm{t}}}$ to $\frac{h_{\mathrm{r}}}{h_{\mathrm{m}}}$. Alternative and/or more concise universal dimensionless functions (i.e., $\Pi_{1}$ to $\Pi_{5}$ ), which fit the same set of data taken from the 76 cases examined in the present study, may also be explored.

\subsection{Forward analysis algorithms}

The forward analysis leads to prediction of the $P-h$ response from known elasto-plastic properties. With the available dimensionless functions $\Pi_{1}, \Pi_{2}$, $\Pi_{3}, \Pi_{4}, \Pi_{5}$ and $\Pi_{6}$, the forward analysis algorithm is readily constructed. One such set of algorithms is shown in Flow Chart 1. Alternatively, $\frac{h_{\mathrm{r}}}{h_{\mathrm{m}}}$ can also be obtained using function $\Pi_{3}$ instead of $\Pi_{4}$. As discussed earlier, $\Pi_{3}$ and $\Pi_{4}$ are interdependent functions.

To verify the accuracy of the proposed algorithms, uniaxial compression and indentation experiments were conducted in two materials: 7075-T651 aluminum and 6061-T6511 aluminum. Values for $E$ and $\sigma_{\mathrm{y}}$ were obtained from the resulting experimental true stress-true total strain data. The value for $\sigma_{0.033}$ was then determined from the true stress-true plastic strain data. Finally, a power law equation was fit to the true stress-true plastic strain data (see Fig. 4) to estimate a value for $n$ (see Table 3). The Poisson's ratio $v$ was not experimentally determined, and was assigned a typical value of 0.33 for aluminum alloys. The parameters $E_{\mathrm{i}}$ and $v_{\mathrm{i}}$ were assigned values of $1100 \mathrm{GPa}$ and 0.07 , respectively; these are typical values for diamond taken from the literature [34]. Microhardness specimens were prepared identically to the microindentation specimens, and were indented on a commercial microhardness tester to a maximum load of $0.1 \mathrm{kgf}$ over a total test time of $20 \mathrm{~s}$. Vickers hardness was calculated as $\mathrm{HV}=1.8544 P / D^{2}$, where $P$ is load (in kgf) and $D$ is the average length of the indentation diagonals (in $\mathrm{mm}$ ) as observed under an

Flow Chart 1: Forward Analysis Algorithms

$$
\text { Forward Problem: } E, n, \sigma_{\mathrm{y}}, v \underset{\begin{array}{l}
\text { set } P_{\mathrm{m}} \\
\text { or } h_{\mathrm{m}}
\end{array}}{\longrightarrow} C, h_{\mathrm{r}}, p_{\mathrm{ave}},\left.\frac{d P_{\mathrm{u}}}{d h}\right|_{h_{\mathrm{m}}}, \frac{W_{\mathrm{p}}}{W_{\mathrm{t}}}
$$

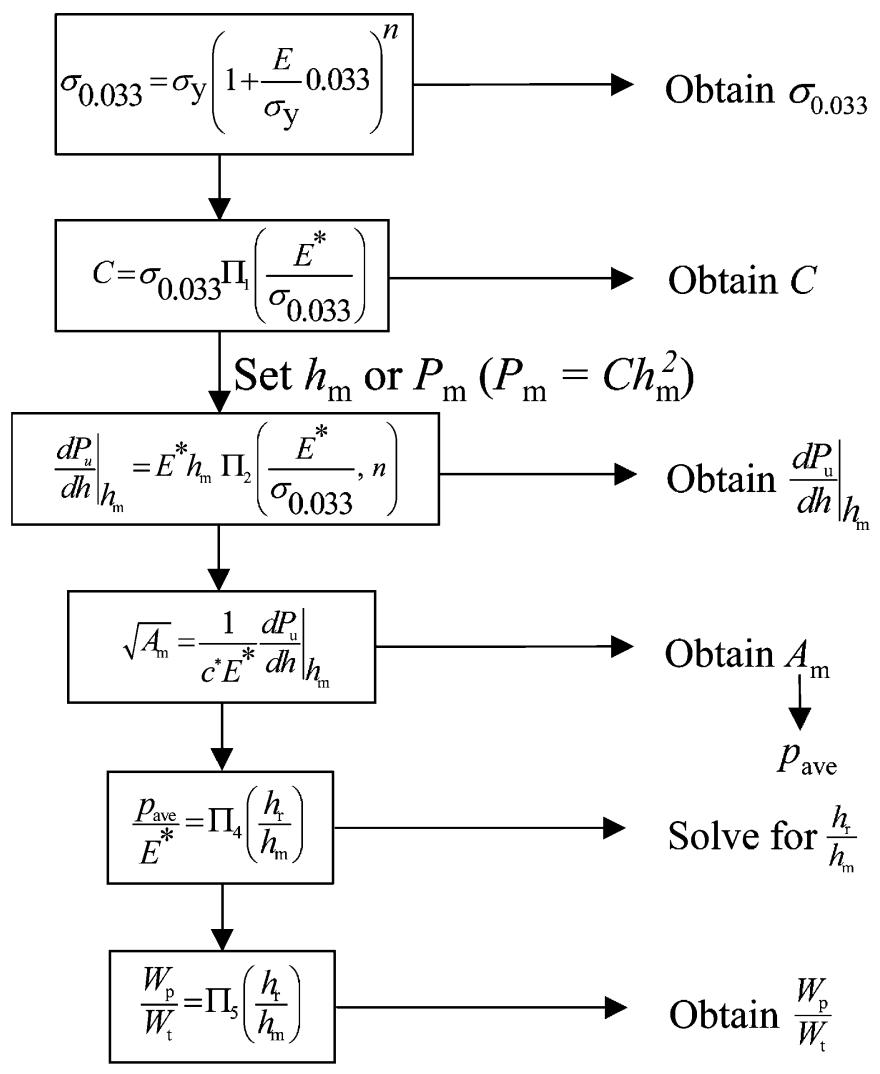


Table 3. Mechanical property values used in the forward analysis

\begin{tabular}{|c|c|c|c|c|c|c|c|c|}
\hline Material & $E(\mathrm{GPa})$ & $v$ & $E^{*}(\mathrm{GPa})$ & $\sigma_{\mathrm{y}}(\mathrm{MPa})$ & $\sigma_{0.033}(\mathrm{MPa})$ & $\begin{array}{l}n \text { from power } \\
\text { law fit }\end{array}$ & $\begin{array}{l}\text { Vickers } \\
\text { hardness }\end{array}$ & $p_{\text {ave }}(\mathrm{MPa})$ \\
\hline Al 6061-T6511 & 66.8 & 0.33 & $70.2^{\mathrm{a}}$ & $284^{\mathrm{b}}$ & 338 & 0.08 & $104.7^{\mathrm{c}}$ & $1108^{\mathrm{d}}$ \\
\hline Al 7075-T651 & 70.1 & 0.33 & $73.4^{\mathrm{a}}$ & $500^{\mathrm{b}}$ & 617.5 & 0.122 & $174.1^{\mathrm{c}}$ & $1842^{\mathrm{d}}$ \\
\hline
\end{tabular}

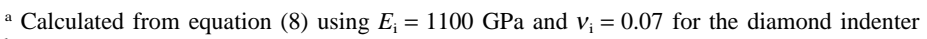

${ }^{\mathrm{b}}$ Estimated at $0 \%$ offset strain

${ }^{c}$ Averaged from 10 and 5 hardness tests $(P=0.1 \mathrm{kgf})$ for Al 6061-T6511 and Al 7075-T651 specimens, respectively

${ }^{\mathrm{d}}$ Estimated from the hardness number assuming that changes in impression size during unloading can be ignored

optical microscope with a $40 \times$ objective lens. The algorithm shown in Flow Chart 1 was applied to solve for $C, \frac{h_{\mathrm{r}}}{h_{\mathrm{m}}}, A_{\mathrm{m}}, p_{\text {ave }}$ and $\left.\frac{\mathrm{d} P_{\mathrm{u}}}{\mathrm{d} h}\right|_{h_{\mathrm{m}}}$. Table 3 lists the mechanical property values used in the forward analysis. Table 4(a) and (b) list the predictions from the forward analysis, along with the values extracted from the experimental indentation data for 7075-T651 aluminum and 6061-T6511 aluminum specimens, respectively. As proposed in [5], the experimental values of $\left.\frac{\mathrm{d} P_{\mathrm{u}}}{\mathrm{d} h}\right|_{h_{\mathrm{m}}}$ listed in Table 4 were obtained by first fitting a power law function $P_{\mathrm{u}}=A\left(h-h_{\mathrm{r}}\right)^{m}$ to $67 \%$ of the unloading data and then evaluating the derivative at $h=h_{m}$. From Table 4 , it is evident that the present forward analysis results are in good agreement with the experimental $P-h$ curves.

\subsection{Reverse analysis algorithms}

The reverse analysis implies estimation of the elasto-plastic properties from one complete (i.e., loading and full unloading) $P-h$ curve. In a similar manner, the dimensionless functions $\Pi_{1}, \Pi_{2}, \Pi_{3}, \Pi_{4}, \Pi_{5}$ and $\Pi_{6}$ allow us to construct the reverse algorithms. A set of the reverse analysis algorithms is shown in Flow Chart 2. Alternatively, due to the interdependence between $\Pi_{3}$ and $\Pi_{4}$, the dimensionless function $\Pi_{3}$ can be used instead of $\Pi_{4}$ to solve the reverse problem, although this alternative set of algorithms involving $\Pi_{3}$ is not as straightforward as that proposed in Flow Chart 2. For those experiments for

Table 4. Forward analysis results on Al 6061-T6511 and Al 7075-T651 (max. load =3 N)

\begin{tabular}{|c|c|c|c|c|c|c|}
\hline & $C(\mathrm{GPa})$ & $\%$ err $C^{\mathrm{a}}$ & $\left.\frac{\mathrm{d} P_{u}}{\mathrm{~d} h}\right|_{h_{m}}(\mathrm{kN} / \mathrm{m})$ & $\left.\% \operatorname{err} \frac{\mathrm{d} P_{u}}{\mathrm{~d} h}\right|_{h_{m}}$ & $W_{\mathrm{p}} / W_{\mathrm{t}}$ & $\%$ err $W_{\mathrm{p}} / W_{\mathrm{t}}$ \\
\hline \multicolumn{7}{|l|}{ (a) Al 6061-T6511 } \\
\hline Test 1 & 27.4 & $-1.6 \%$ & 4768 & $1.6 \%$ & 0.902 & $0.8 \%$ \\
\hline Test 2 & 28.2 & $1.2 \%$ & 4800 & $2.3 \%$ & 0.905 & $1.2 \%$ \\
\hline Test 3 & 27.2 & $-2.4 \%$ & 4794 & $2.2 \%$ & 0.904 & $1.1 \%$ \\
\hline Test 4 & 27.3 & $-2.2 \%$ & 4671 & $-0.4 \%$ & 0.889 & $-0.6 \%$ \\
\hline Test 5 & 27.0 & $-3.2 \%$ & 4762 & $1.5 \%$ & 0.889 & $-0.6 \%$ \\
\hline Test 6 & 27.6 & $-0.9 \%$ & 4491 & $-4.2 \%$ & 0.891 & $-0.4 \%$ \\
\hline Ave & 27.4 & & 4715 & & 0.896 & \\
\hline$S T D E V^{\mathrm{b}}$ & 0.6 & & 110.9 & & 0.007 & \\
\hline$S T D E V / X_{\text {prediction }}$ & $2.1 \%$ & & $2.4 \%$ & & $0.8 \%$ & \\
\hline $\begin{array}{l}\text { Forward prediction } \\
\text { (assume } v=0.33 \\
\text { and Berkovich } c^{*} \text { ) }\end{array}$ & 27.9 & & 4691 & & 0.894 & \\
\hline \multicolumn{7}{|l|}{ (b) Al 7075-T651 } \\
\hline Test 1 & 42.0 & $-4.2 \%$ & 3665 & $2.2 \%$ & 0.833 & $1.0 \%$ \\
\hline Test 2 & 40.9 & $-6.9 \%$ & 3658 & $2.1 \%$ & 0.838 & $1.7 \%$ \\
\hline Test 3 & 42.3 & $-3.7 \%$ & 3654 & $1.9 \%$ & 0.832 & $1.0 \%$ \\
\hline Test 4 & 43.1 & $-1.7 \%$ & 3744 & $4.5 \%$ & 0.836 & $1.5 \%$ \\
\hline Test 5 & 43.5 & $-0.7 \%$ & 3789 & $5.7 \%$ & 0.839 & $1.8 \%$ \\
\hline Test 6 & 44.6 & $1.6 \%$ & 3706 & $3.4 \%$ & 0.831 & $0.9 \%$ \\
\hline Ave & 42.7 & & 3703 & & 0.835 & \\
\hline$S T D E V^{\mathrm{b}}$ & 1.6 & & 128.1 & & 0.011 & \\
\hline$S T D E V / X_{\text {prediction }}$ & $3.7 \%$ & & $3.6 \%$ & & $1.3 \%$ & \\
\hline $\begin{array}{l}\text { Forward prediction } \\
\text { (assume } v=0.33 \\
\text { and Berkovich } c^{*} \text { ) }\end{array}$ & 43.9 & & 3585 & & 0.824 & \\
\hline
\end{tabular}

a All errors were computed as $\frac{X_{\text {test }}-X_{\text {prediction }}}{X_{\text {prediction }}}$, where $X$ represents a variable

b $S T D E V=\sqrt{\frac{1}{N} \sum_{i=1}^{N}\left(X_{\text {test }}-X_{\text {prediction }}\right)^{2}}$, where $X$ represents a variable 
which it is difficult to measure $h_{\mathrm{r}}$ accurately, as proposed by Giannakopoulos and Suresh [20], one can measure $\frac{W_{\mathrm{p}}}{W_{\mathrm{t}}}$ instead and use the dimensionless function $\Pi_{5}$ to obtain $\frac{h_{\mathrm{r}}}{h_{\mathrm{m}}}$.

To verify the reverse analysis algorithms, twelve experimental $P-h$ curves (six from 6061-T6511 aluminum specimens and six from 7075-T651 aluminum specimens) shown in Table 4 were analyzed to extract elasto-plastic properties of the indented specimens; results are shown in Table 5. From Table 5, it is clear that the proposed reverse algorithms yield accurate estimates of $E$ and $\sigma_{0.033}$, and give reasonable estimates of $\sigma_{\mathrm{y}}$ (especially after taking an average from the six indentation results) which agree well with experimental compression data. It is noted that changing the definition of $\sigma_{\mathrm{y}}$ to $0.1 \%$ or $0.2 \%$ (instead of $0 \%$ ) offset strain would not affect the main conclusions. The average pressure $p_{\text {ave }}$ also compares well with values estimated from experimental microhardness tests. The fractional errors observed in obtaining $n$ are somewhat misleading, due to the fact that $n \ll 1$. Although the errors obtained from individual $P-h$ curves were relatively large in a few cases, the values averaged from a number of indentation tests appeared to be more reliable. Results in Table 5 also show that the proposed reverse algorithms give better predictions than Oliver and Pharr [5] and Doerner and Nix [3] methods for extracting $E^{*}$ values. This improved calculation of elastic properties is likely due to the fact that sink-in/pile-up effects were taken into account with present model.

\section{DISCUSSION OF UNIQUENESS, SENSITIVITY AND REPRESENTATIVE STRAINS}

\subsection{Forward analysis and reverse analysis}

In order to verify the proposed forward algorithms shown in Flow Chart 1, computational results from the 76 sets of elasto-plastic parameters were taken as input to predict the $P-h$ responses. Each of the forward analyses resulted in a single set of output $\left(C, \frac{h_{\mathrm{r}}}{h_{\mathrm{m}}}\right.$ and $\left.\left.\frac{\mathrm{d} P_{\mathrm{u}}}{\mathrm{d} h}\right|_{h_{\mathrm{m}}}\right)$ which agreed well with the FEMpredicted $P-h$ response.

Similarly for the reverse problem, the 76 cases of the forward analysis (output) results were used as input to verify the reversibility of the reverse analysis algorithms proposed in Flow Chart 2. In only two cases, $\left(\frac{\sigma_{\mathrm{y}}}{E}, n\right)=(0.033,0.5)$ and $(0.04,0.5)$ with very high hardening $(n=0.5)$, the reverse analysis yielded two solutions of $n$ ( $E^{*}$ and $\sigma_{\mathrm{r}}$ still gave the correct answers). Reverse analysis on the remaining 74 cases resulted in a single, accurate re-construction of the initial elasto-plastic parameters.

As established in the literature (e.g., $[5,12,16])$, $E^{*}$ may be uniquely obtained from a single $P-h$ curve via equation (23). Alternatively, noting that $p_{\text {ave }}=\frac{P_{\mathrm{m}}}{A_{\mathrm{m}}}$, it is readily shown that dimensionless functions $\Pi_{4}$ and $\Pi_{6}$ can be combined to solve for $E^{*}$ and $A_{\mathrm{m}}$. From known $E^{*}$, the dimensionless function $\Pi_{1}$ can be used to determine the value of $\sigma_{0.033}$ [see Fig. $8(\mathrm{~b})]$. Consequently, after both $E^{*}$ and $\sigma_{0.033}$ are

Flow Chart 2: Reverse Analysis Algorithms

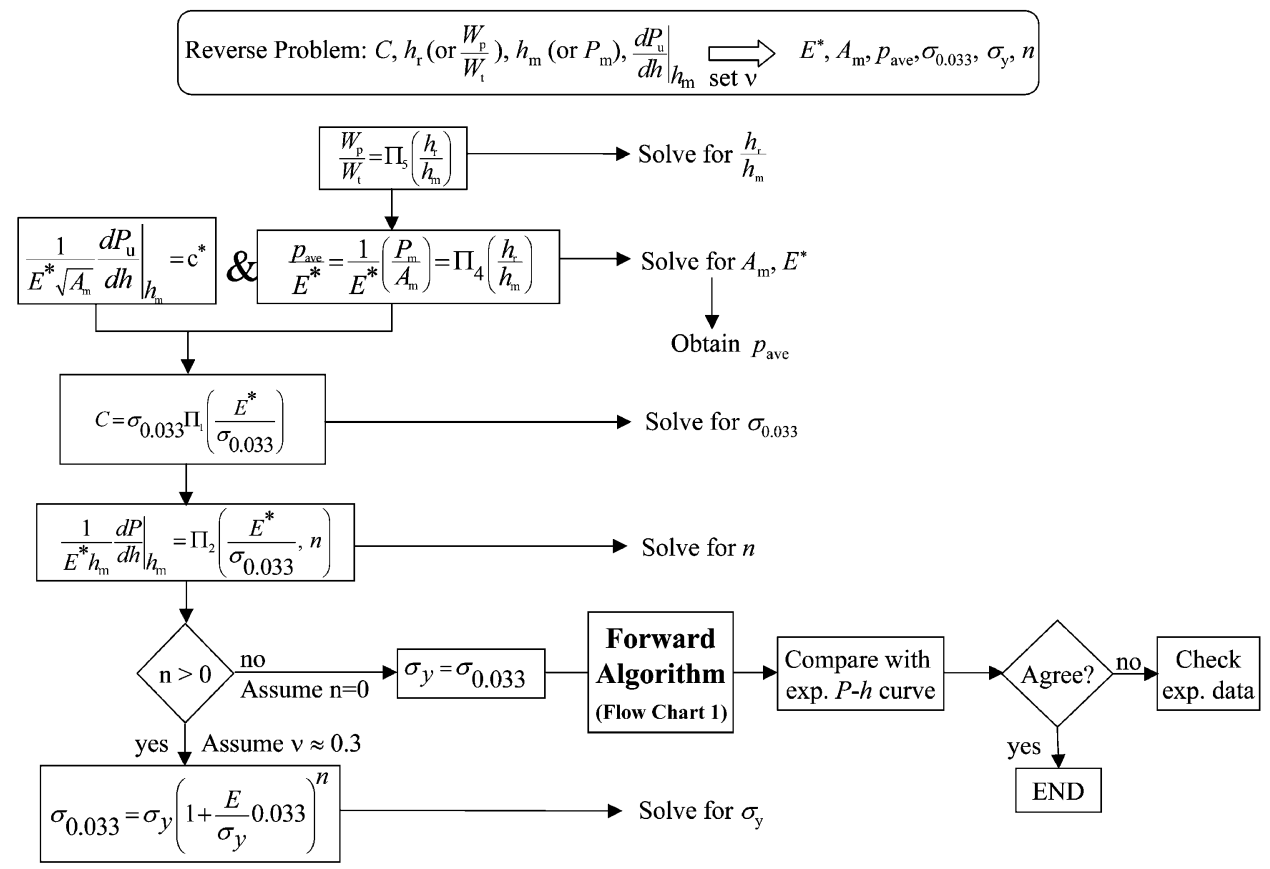




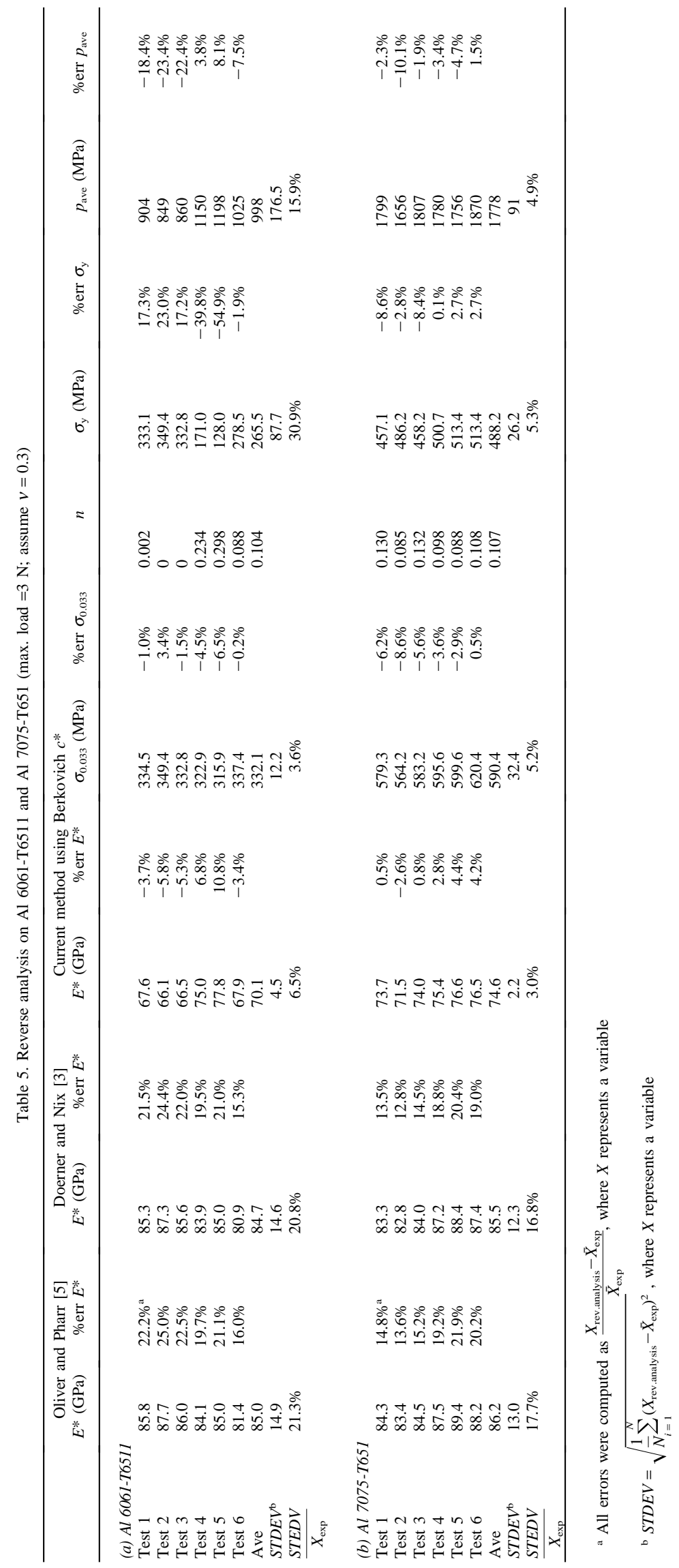


determined, strain hardening exponent $n$ can be determined by dimensionless function $\Pi_{2}$ or $\Pi_{3}$ [see Fig. 10(a) and (b)]. It was found in the current study that, when the assumptions of the model are valid, a single value for $E^{*}$ and $\sigma_{0.033}$ can be determined for all cases. Furthermore, except for cases where $\frac{\sigma_{y}}{E} \geq$ 0.033 and $n>0.3$, one single value of $n$ can be determined as well (although both $\sigma_{\mathrm{y}}$ and $n$ are highly sensitive to even small variations in $P-h$ response). Examining Fig. 10(a) in detail, when $\frac{\sigma_{y}}{E} \geq$ $0.033\left(\frac{\sigma_{y}}{E^{*}} \geq 0.03\right)$ and $n>0.3$, the $n=0.5$ curve crossed the other three curves, which indicates the solution of $n$ may not be unique using dimensionless function $\Pi_{2}$ in that region; a single solution of $n$ may be obtained using dimensionless function $\Pi_{3}$ instead. The above arguments regarding uniqueness can only be valid when the $P-h$ responses can be measured accurately and precisely. Therefore, with accurate $P-h$ curves, the uniqueness of the reverse problem can be ensured for low hardening materials (i.e., $n \leq 0.3$ ); the uniqueness can also be preserved if $\frac{\sigma_{y}}{E^{*}}<0.03$ for higher hardening materials (i.e., $0.3<$ $n \leq 0.5)$. Considering the fact that each of the dimensionless functions $\Pi_{1}, \Pi_{2}, \ldots$, and $\Pi_{6}$ carries a small amount of uncertainty, definitive conclusions can not be drawn as yet regarding the uniqueness of the reverse analysis when $\frac{\sigma_{y}}{E^{*}} \geq 0.03$ and $0.3<n \leq 0.5$. Table 6 summarizes the above-mentioned results.

Cheng and Cheng [19] examined whether uniaxial stress-strain relationships of materials can be uniquely determined by matching the loading and unloading $P-h$ curves, calculated using their FEM analysis and scaling relationships, with those measured experimentally. By showing that there could be multiple stress-strain curves for a given set of loading and unloading curves, they concluded that the material stress-strain behavior may not be uniquely determined from the loading and unloading $P-h$ response alone. We examined all seven cases presented in [19]. For the four cases presented in Fig.

Table 6. Uniqueness of reverse analysis

\begin{tabular}{lll}
\hline Mechanical property & Solution unique? \\
\hline$E^{*}$ & & Yes \\
Hardness $\left(p_{\text {ave }}\right)$ & Yes \\
$\sigma_{\mathrm{r}}$ & Yes \\
$\sigma_{\mathrm{y}}$ & Yes \\
& when $n \leq 0.3$ & Yes \\
& and $\frac{\sigma_{\mathrm{y}}}{E^{*}}<0.03$ & \\
& when $0.3<n \leq 0.5$ & $?$ \\
& and $\frac{\sigma_{\mathrm{y}}}{E^{*}} \geq 0.03$ & \\
\hline
\end{tabular}

3(a) of [19], the values of $\frac{\sigma_{y}}{E^{*}}$ are beyond the range of the current study. That is, the ratio of $\frac{\sigma_{\mathrm{y}}}{E_{*}}$ given by these four cases $\left(\sim 10^{-1}\right)$ may not accurately describe any metallic engineering alloys (see, e.g., [35]), but may describe certain ceramics or engineering polymers which are not well-described by power law plasticity. Therefore, the non-uniqueness of these cases is physically irrelevant to the scope of our analysis. In contrast, the cases reported in Fig. 3(b) of [19] are within our range of parameters $\left(\frac{\sigma_{\mathrm{y}}}{E^{*}} \sim 10^{-2}\right.$ to $\left.10^{-3}\right)$. The current forward analysis predicts three $P-h$ responses which are statistically unique in terms of the calculated curve parameters such as $C, \frac{h_{\mathrm{r}}}{h_{\mathrm{m}}}$ and $\left.\frac{\mathrm{d} P_{\mathrm{u}}}{\mathrm{d} h}\right|_{h_{\mathrm{m}}}$. This statistical uniqueness does not directly contradict Cheng and Cheng's assertion of nonuniqueness, as they used a different apex angle in their FEM simulations, and as the $P-h$ curves appear visually similar. The maximum variation in curve parameters calculated by our forward analysis of these three cases was an $8 \%$ change in $\left.\frac{\mathrm{d} P_{\mathrm{u}}}{\mathrm{d} h}\right|_{h_{\mathrm{m}}}$. The present reverse analysis provides a unique solution in that, even if the calculated loading curvatures $(C)$ were mathematically identical for two separate $P-h$ responses, small variations in terms of $\left.\frac{\mathrm{d} P_{\mathrm{u}}}{\mathrm{d} h}\right|_{h_{m}}$ or $\frac{h_{\mathrm{r}}}{h_{\mathrm{m}}}$ are sufficient to calculate a unique value of $n$ and, consequently, a unique value of $\sigma_{\mathrm{y}}$ for each case. However, these small variations in curve parameters may not be visually apparent when plotting these $P-h$ responses simultaneously. In fact, as experimental scatter may cause such variation in $P-h$ curve parameters, the issue of sensitivity in these analyses is an important consideration.

\subsection{Sensitivity to forward analysis, reverse analysis and apex angle}

For forward analysis, the sensitivity of the predicted $P-h$ response parameters to variations in the input mechanical properties of the indented material was investigated for the 76 cases examined in this study. The results showed that a $\pm 5 \%$ change in any one input parameter (i.e., $E^{*}, \sigma_{\mathrm{y}}$ or $n$ ), would lead to variations of less than $\pm 6 \%$ in the predicted results $\left(C, \frac{h_{\mathrm{r}}}{h_{\mathrm{m}}}\right.$ and $\left.\left.\frac{\mathrm{d} P_{\mathrm{u}}}{\mathrm{d} h}\right|_{h_{\mathrm{m}}}\right)$.

As discussed in Venkatesh et al. [21], the accuracy with which the mechanical properties of the indented material can be estimated through reverse analysis could depend strongly on the accuracy with which the $P-h$ responses are measured. The sensitivity of 
the estimated mechanical properties to variations in the input parameters obtained from the $P-h$ curves was investigated for the 76 cases examined in this study as well. For each of these cases, the sensitivity of the estimated elasto-plastic properties to variations in the three $P-h$ curve parameters $-C,\left.\frac{\mathrm{d} P_{\mathrm{u}}}{\mathrm{d} h}\right|_{h_{\mathrm{m}}}$, and $\frac{W_{\mathrm{p}}}{W_{\mathrm{t}}}$-about their respective reference values (as estimated from the forward analysis) was examined. The results are summarized in Table 7 . In general, sensitivity to reverse analysis is different for each individual case, thus the maximum variations listed in Table 7 are conservative estimates.

It is evident that $E^{*}$ displayed weak sensitivity with respect to $C$ and $\left.\frac{\mathrm{d} P_{\mathrm{u}}}{\mathrm{d} h}\right|_{h_{\mathrm{m}}}$, and moderate sensitivity to $\frac{W_{\mathrm{p}}}{W_{\mathrm{t}}} ; \sigma_{\mathrm{r}}$ displayed weak sensitivity with respect to $\frac{W_{\mathrm{p}}}{W_{\mathrm{t}}}$, and moderate sensitivity to both $C$ and $\left.\frac{d \mathrm{P}_{\mathrm{u}}}{\mathrm{d} h}\right|_{h_{\mathrm{m}}}$; for low hardening materials $(n \leq 0.1), \sigma_{\mathrm{y}}$ displayed moderate sensitivity to $C$ and $\left.\frac{\mathrm{d} P_{\mathrm{u}}}{\mathrm{d} h}\right|_{h_{\mathrm{m}}}$, and strong sensitivity to $\frac{W_{\mathrm{p}}}{W_{\mathrm{t}}}$; for higher hardening materials $(n>0.1), \sigma_{\mathrm{y}}$ displayed strong sensitivity to all three parameters; $p_{\text {ave }}$ displayed weak sensitivity to $C$ and $\left.\frac{\mathrm{d} P_{\mathrm{u}}}{\mathrm{d} h}\right|_{h_{\mathrm{m}}}$, and moderate sensitivity to $\frac{W_{\mathrm{p}}}{W_{\mathrm{t}}}$. The results of the reverse sensitivity analysis shown in Table 7 are consistent with the reverse analyses of experimental $P-h$ curves listed in Table 5. The greater scatter in computed $\sigma_{\mathrm{y}}$ values in Table 5 reflects the stronger sensitivity with respect to $\sigma_{\mathrm{y}}$. If data scatter is random in nature, it is expected that taking the averaged value from a number of indentation tests may significantly reduce the error, as clearly demonstrated in Table 5.

In addition, the sensitivity of the $P-h$ response as computed from FEM calculations to variations in the apex angle $\theta$ of the indenter was investigated for the four cases A, B, C and D listed in Table 1. The results are summarized in Table 8 and Fig. 12, where the respective reference values (as calculated from the FEM computed $P-h$ curves) were taken at $\theta=70.3^{\circ}$. From Fig. 12 and Table 8, the dependence of $C,\left.\frac{\mathrm{d} P_{\mathrm{u}}}{\mathrm{d} h}\right|_{h_{\mathrm{m}}}$ and $\frac{W_{\mathrm{p}}}{W_{\mathrm{t}}}$ to variations in the apex angle appears to be quite significant and approximately linear. Taking $\theta=68^{\circ}$ as used in $[11,12,19]$ as an example, deviation from the reference apex angle by $2.3^{\circ}$ results in maximum variations of $-20 \%$, $-11.7 \%$ and $+5.7 \%$ in terms of $C,\left.\frac{\mathrm{d} P_{\mathrm{u}}}{\mathrm{d} h}\right|_{h_{\mathrm{m}}}$ and $\frac{W_{\mathrm{p}}}{W_{\mathrm{t}}}$ respectively (see Table 8). As evident in Table 7, variations of this magnitude are beyond the error tolerance limit of reverse analysis. The universal functions based on a $68^{\circ}$ apex angle may significantly differ from those obtained using a $70.3^{\circ}$ apex angle, although certain trends with respect to various parameters may be similar. Commercially available diamond indenters are normally within $\pm 0.5^{\circ}$ of the specified apex angle. The resulting maximum variations in $P-h$ curve parameters as estimated from FEM computations are around $\pm 3 \%, \pm 3 \%$, and $\pm 1 \%$ in terms of $C,\left.\frac{\mathrm{d} P_{\mathrm{u}}}{\mathrm{d} h}\right|_{h_{\mathrm{m}}}$ and $\frac{W_{\mathrm{p}}}{W_{\mathrm{t}}}$ respectively, which are within the error tolerance limit of the reverse analysis.

\subsection{Representative strains}

The concept of representative strain was first introduced by Tabor [1] to relate its corresponding representative stress to the hardness value. Tabor [1] suggested a representative plastic strain of $8-10 \%$ based on experimental observations. This original definition does not represent any apparent physical transition in mechanical response. Giannakopoulos et al. [18] and Giannakopoulos and Suresh [20] used a "characteristic strain" of 29-30\% within the context of equation (20). Giannakopoulos and Suresh [20] suggested that the region of material experiencing strains beyond $29 \%$ under the indenter exhibits plastic "cutting" characteristics and may be modeled using slip line theory. In the current study, a representative plastic strain $\varepsilon_{\mathrm{r}}=0.033$ was identified as a strain level which allows for the construction of a dimensionless description of the indentation loading response [i.e., equation (11b)], independent of strain hardening exponent $n$. Here, the underlying connections between these three different definitions and the corresponding representative strain levels are discussed.

To understand these different representative strain values, it is important to note that the dimensionless function $\Pi_{1}$ in Fig. 8(b), used to identify $\varepsilon_{\mathrm{r}}=0.033$ has a different functional form than that used by the earlier researchers, e.g., equation (20). Therefore, it is possible for these different definitions to result in different solutions. Equation (21) is a modification of equation (20) and, as mentioned in Section 2.5, can fit all 76 cases studied reasonably well. For low strain hardening materials, $\sigma_{0.29} \approx \sigma_{\mathrm{r}}\left(\approx \sigma_{\mathrm{y}}\right)$, and thus equation (21) can be rewritten as

$$
\begin{gathered}
C \approx N_{1} \sigma_{0.29}\left(1+\frac{\sigma_{\mathrm{y}}}{\sigma_{0.29}}\right)\left[N_{2}+\ln \left(\frac{E^{*}}{\sigma_{\mathrm{r}}}\right)\right] \\
=\left(\frac{\sigma_{\mathrm{y}}+\sigma_{0.29}}{2}\right)\left\{2 N_{1}\left[N_{2}+\ln \left(\frac{E^{*}}{\sigma_{\mathrm{r}}}\right)\right]\right\} \\
=\left(\frac{\sigma_{\mathrm{y}}+\sigma_{0.29}}{2}\right) \Pi_{1 \mathrm{~N}}\left(\frac{E^{*}}{\sigma_{\mathrm{r}}}\right)
\end{gathered}
$$

within a $\pm 5.5 \%$ error of equation (21) for all the 76 cases computed in the current study; $\Pi_{1 \mathrm{~N}}$ is a dimen- 


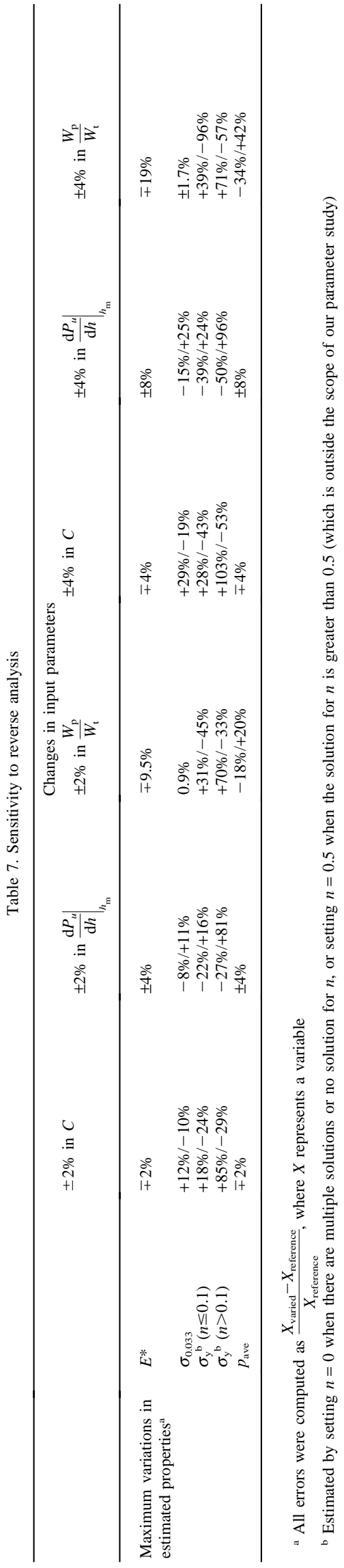


Table 8. Apex angle sensitivity of A, B, C, and D four cases

\begin{tabular}{|c|c|c|c|c|}
\hline $\begin{array}{c}\text { Maximum / Apex } \\
\text { variations } / \text { angle }\end{array}$ & $68^{\circ}$ & $69^{\circ}$ & $70.3^{\circ}$ (reference) & $72^{\circ}$ \\
\hline$\Delta C / C_{\mathrm{ref}}$ & $-20.4 \%$ & $-12.1 \%$ & $0 \%$ & $+19.3 \%$ \\
\hline$\Delta\left(\frac{\mathrm{d} P}{\mathrm{~d} h}\right) /\left(\frac{\mathrm{d} P}{\mathrm{~d} h}\right)_{\text {ref }}$ & $-11.7 \%$ & $-7.7 \%$ & $0 \%$ & $+9.0 \%$ \\
\hline$\Delta\left(\frac{W_{\mathrm{p}}}{W_{\mathrm{t}}}\right) /\left(\frac{W_{\mathrm{p}}}{W_{\mathrm{t}}}\right)_{\text {ref }}$ & $+5.7 \%$ & $+3.0 \%$ & $0 \%$ & $-4.8 \%$ \\
\hline
\end{tabular}

(a)

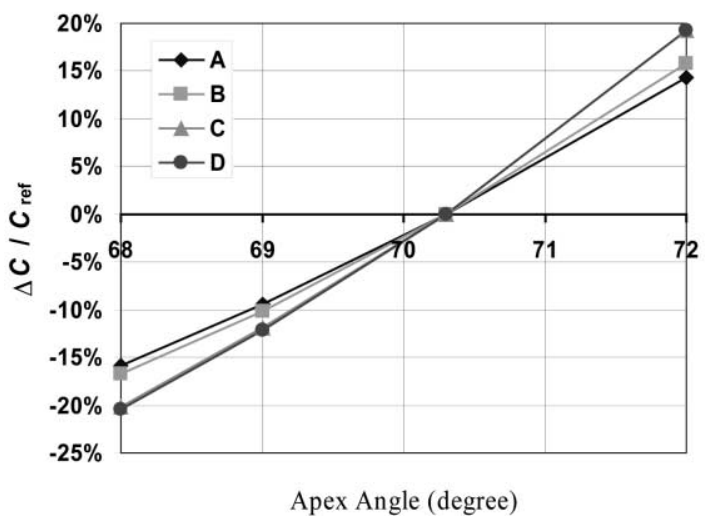

(b)

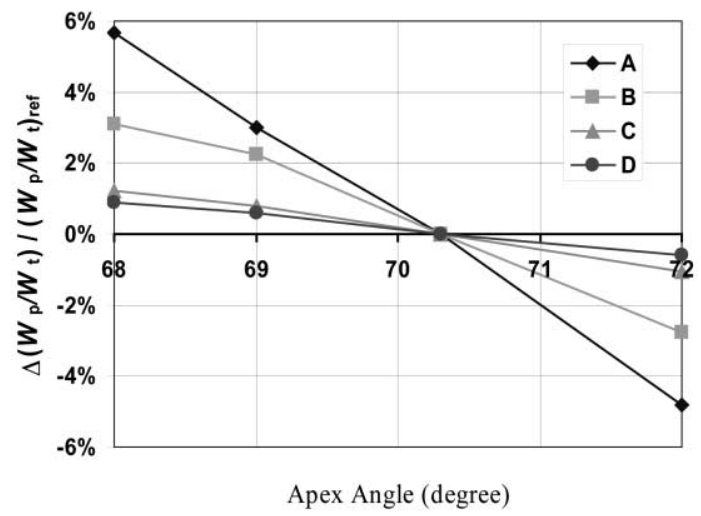

Fig. 12. Sensitivity of (a) loading curvature $C$ and (b) plastic work ratio $\frac{W_{\mathrm{p}}}{W_{\mathrm{t}}}$ to variations in apex angle for four model materials. A two-degree variation in apex angle resulted in 15-20\% variations in loading curvature $C$ and up to $6 \%$ variations in plastic work ratio $\frac{W_{\mathrm{p}}}{W_{\mathrm{t}}}$.

sionless function. One can solve for $\varepsilon_{\mathrm{r}}$ that gives $\sigma_{\mathrm{r}}$ as an arithmetic average between $\sigma_{\mathrm{y}}$ and $\sigma_{0.29}$ :

$$
\sigma_{\mathrm{r}}=\frac{\sigma_{\mathrm{y}}+\sigma_{0.29}}{2}
$$

If it is assumed that $\left.\sigma_{y} \approx \sigma\right|_{\varepsilon=0.002}$, then equation (27) becomes

$$
R \varepsilon_{\mathrm{r}}^{n} \approx \frac{R \cdot 0.002^{n}+R \cdot 0.29^{n}}{2}
$$

and thus

$$
\varepsilon_{\mathrm{r}} \approx\left(\frac{0.002^{n}+0.29^{n}}{2}\right)^{\frac{1}{n}}
$$

For low hardening materials with $0<n<0.15$, equation (29) gives $0.024<\varepsilon_{\mathrm{r}}<0.038$, which is fairly close to 0.033. This exercise indicates that: (1) $\varepsilon_{\mathrm{r}}=0.033$ obtained in the current study is fundamentally linked to the "characteristic strain" of 0.29 given in the literature, and the differences in the magnitude of the strain come from their different functional definitions; (2) $\sigma_{0.033}$ taken at the 0.033 representative strain is a "weighted average" of the stresses over the range between $\varepsilon_{\mathrm{y}}$ and $\varepsilon_{\mathrm{p}}=0.29$. Additional computations confirmed that stress-strain behavior beyond $\varepsilon_{\mathrm{p}}=0.29$ has little effect on a material's $P-h$ response.

Earlier studies by Tabor [1] and Johnson [15] gave a representative strain at about $\varepsilon_{\mathrm{r}}=0.08$. This estimation relied on experimental data which indicated that

$$
\frac{H}{\sigma_{\mathrm{r}}}=\frac{p_{\mathrm{ave}}}{\sigma_{\mathrm{r}}} \approx 3.0
$$

where $H$ is the hardness expressed in units of pressure. Again, it is noted that the functional form defined by equation (30) is different from those used to define representative strains of $3.3 \%$ or $29 \%$, and it is natural that different functions may lead to different results. To better understand this problem, a new dimensionless function can be defined

$$
\frac{p_{\text {ave }}}{\sigma_{\mathrm{r}}}=\Pi_{7}\left(\frac{\sigma_{\mathrm{r}}}{E^{*}}, n\right)
$$


Similar to the procedure taken to obtain $\varepsilon_{\mathrm{r}}=0.033$ (see Fig. 8), errors were minimized using the least squares method to fit results obtained from the 76 cases studied. It was found that when $\varepsilon_{\mathrm{r}}=0.082$, a best fit function within a $\pm 5.96 \%$ error was achieved with the following $n$-independent function

$$
\begin{gathered}
\Pi_{7}=\frac{p_{\mathrm{ave}}}{\sigma_{0.082}} \approx-15.4944\left(\frac{\sigma_{0.082}}{E^{*}}\right)^{2} \\
-15.1699\left(\frac{\sigma_{0.082}}{E^{*}}\right)+2.7497
\end{gathered}
$$

A representative strain of $\varepsilon_{\mathrm{r}}=0.082$ was thus identified. Figure 13 shows equation (32) plotted against the FEM data. Also plotted in Fig. 13 are the four cases studied in Section 2.5. Equation (32) predicts $\Pi_{7}=\frac{p_{\text {ave }}}{\sigma_{0.082}} \approx 2.75$ for both steel and aluminum listed in Table 1. It is noted that, in Tabor's original study [1], when there was no pre-straining, $\frac{p_{\text {ave }}}{\sigma_{0.08}}$ was found to be 2.6 and 2.84 (instead of being very close to 3.0) for annealed copper and mild steel, respectively; this is in good agreement with the predictions from $\Pi_{7}$.

Although different functional definitions were used by Tabor and the present study, the same set of modeling assumptions and fitting algorithms were used to derive a characteristic strain of $8.2 \%$. This exercise shows that these two values are fundamentally linked to one another. Thus, the apparent disparity comes not from discrepancies in data, constitutive modeling, or fitting procedures, but rather from the choice of functional definitions that were used to relate certain indentation parameters to certain mechanical properties.

\section{CONCLUSIONS}

In this study, dimensional analysis and large deformation finite element studies were performed to eluci-

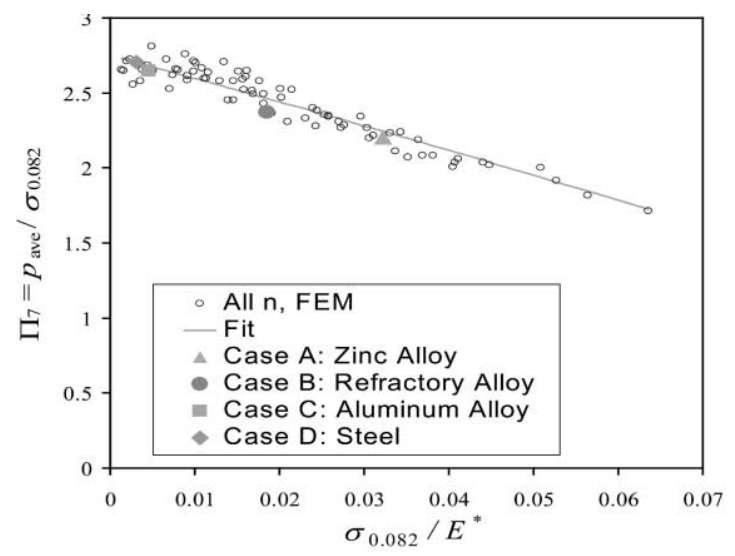

Fig. 13. Dimensionless function $\Pi_{7}$. A best fit function within a $\pm 5.96 \%$ error was achieved with a representative plastic strain $\varepsilon_{\mathrm{r}}=0.082$ and its corresponding stress $\sigma_{0.082}$. date the mechanics of instrumented sharp indentation. Systematic experiments were conducted to verify the theoretical results. The key results of this investigation can be summarized as follows.

1. Using dimensional analysis, a set of new universal, dimensionless functions were constructed to characterize instrumented sharp indentation. From these functions and elasto-plastic finite element computations, solutions were formulated to relate indentation data to elasto-plastic properties.

2. Forward and reverse analysis algorithms were established based on the identified dimensionless functions. These algorithms allow for the calculation of the indentation response for a given set of properties, and also for extraction of some elastoplastic properties from a given set of indentation data, thus obviating the need for large-scale finite element computations after each indentation test.

3 . The proposed forward analysis algorithms work well and robustly; a $\pm 5 \%$ error in any input parameter results in less than $\pm 6 \%$ in the predicted values of $C, \frac{h_{\mathrm{r}}}{h_{\mathrm{m}}}$ or $\left.\frac{\mathrm{d} P_{\mathrm{u}}}{\mathrm{d} h}\right|_{h_{\mathrm{m}}}$. Theoretical predictions compare well with experimental $P-h$ curves.

4. The proposed reverse analysis algorithms were found to predict $E^{*}$ and $\sigma_{0.033}$ quite well, and $\sigma_{\mathrm{y}}$ reasonably well for the few cases studied. Although greater scatter was found in the computation of $\sigma_{y}$, the averaged value approached the experimentally measured yield strength $\sigma_{\mathrm{y}}$. The average pressure $p_{\text {ave }}$ also compared well with values estimated from experimental microhardness tests.

5. Assuming large deformation in FEM simulations and an isotropic power law elasto-plastic constitutive description of the material within the specified range of material parameters, except for cases where $\frac{\sigma_{\mathrm{y}}}{E} \geq 0.033$ and $n>0.3$, the reverse algorithms were able to predict one single set of values for $E^{*}, \sigma_{0.033}$ and $\sigma_{\mathrm{y}}$; furthermore, if the power law assumption holds, the full stress-strain response can be estimated.

6. For sharp indentation of power law hardening pure metals and alloys, a representative strain $\varepsilon_{\mathrm{r}}$ was identified at $3.3 \%$. Within the same theoretical framework, it was demonstrated that the apparent disparities between the value of $3.3 \%$ identified in the current study and the values of $8 \%$ and $29 \%$ proposed in the literature stem from the different functional definitions used to obtain these values, rather than from any intrinsic differences in mechanistic interpretations.

7. A comparative study showed that the $P-h$ responses obtained using large deformation theory exhibited loading curvature $C$ considerably greater 
than those obtained using small deformation theory.

8. Comprehensive sensitivity analyses were carried out for both forward and reverse algorithms. Forward analysis algorithms were found to be accurate and robust. For the sensitivity to reverse analysis, $E^{*}, \sigma_{0.033}$ and $p_{\text {ave }}$ displayed weak or moderate sensitivity to variations in $C,\left.\frac{\mathrm{d} P_{\mathrm{u}}}{\mathrm{d} h}\right|_{h_{\mathrm{m}}}$, and $\frac{W_{\mathrm{p}}}{W_{\mathrm{t}}}$; for low hardening materials $(n \leq 0.1), \sigma_{\mathrm{y}}$ displayed moderate sensitivity to $C$ and $\left.\frac{\mathrm{d} P_{\mathrm{u}}}{\mathrm{d} h}\right|_{h_{\mathrm{m}}}$, and strong sensitivity to $\frac{W_{\mathrm{p}}}{W_{\mathrm{t}}}$; for higher hardening materials $(n>0.1), \sigma_{\mathrm{y}}$ displayed strong sensitivity to all three parameters. Sensitivity of the $P-h$ responses to apex angle deviations were found to be significant with even a $1-2^{\circ}$ deviation; nevertheless, the $P-$ $h$ response variations with respect to apex angle deviations less than $\pm 0.5^{\circ}$ were within the tolerance limit of the reverse analysis.

9. We note that plastic properties of materials extracted from instrumented indentation are very sensitive to even small variations in the $P-h$ responses. Nevertheless, the present computational study provides a mean to determine these plastic properties, which may not be easily obtainable by other means in small volume structures, and further provides an indication of the level of the sensitivity to experimental indentation data.

Acknowledgements - This research was supported by a subcontract to MIT through the Center for Thermal Spray Research at Stony Brook, under the National Science Foundation Grant DMR-0080021, and by the Defense University Research Initiative on Nano-Technology (DURINT) on "Damage and Failure Resistant Nanostructured Materials and Interfacial Coatings" which is funded at MIT by the Office of Naval Research, Grant No. N00014-01-1-0808. KJVV is funded by the National Defense Science and Engineering Graduate Fellowship. The authors gratefully acknowledge helpful and stimulating discussions with Dr A.E. Giannakopoulos and Mr Y.J. Choi during the course of this study.

\section{REFERENCES}

1. Tabor, D., Hardness of Metals. Clarendon Press, Oxford, 1951.

2. Tabor, D., Rev. Phys. Technol., 1970, 1, 145.

3. Doener, M. F. and Nix, W. D., J. Mater. Res., 1986, 1, 601

4. Pharr, G. M. and Cook, R. F., J. Mater. Res., 1990, 5, 847.

5. Oliver, W. C. and Pharr, G. M., J. Mater. Res., 1992, 7, 1564.

6. Field, J. S. and Swain, M. V., J. Mater. Res., 1993, 8, 297.

7. Field, J. S. and Swain, M. V., J. Mater. Res., 1995, 10, 101

8. Gerberich, W. W., Nelson, J. C., Lilleodden, E. T., Anderson, P. and Wyrobek, J. T., Acta mater., 1996, 44, 3585.

9. Bolshakov, A., Oliver, W. C. and Pharr, G. M., J. Mater Res., 1997, 11, 760.

10. Alcala, J., Giannakopoulos, A. E. and Suresh, S., J. Mater. Res., 1998, 13, 1390.
11. Cheng, Y. T. and Cheng, C. M., J. Appl. Phys., 1998, 84, 1284.

12. Cheng, Y. T. and Cheng, C. M., Appl. Phys. Lett., 1998, 73, 614.

13. Suresh, S., Nieh, T. -G. and Choi, B. W., Scripta mater., 1999, 41, 951.

14. Gouldstone, A., Koh, H. -J., Zeng, K. -Y., Giannakopoulos, A. E. and Suresh, S., Acta mater., 2000, 48, 2277.

15. Johnson, K. L., J. Mech. Phys. Solids, 1970, 18, 115.

16. Suresh, S., Alcala, J. and Giannakopoulos, A. E., US Patent No. 6,134,954, October 24, 2000.

17. Dao, M., Chollacoop, N., Van Vliet, K. J., Venkatesh, T. A. and Suresh S., US Provisional Patent, filed with the US Patent Office on March 7, 2001.

18. Giannakopoulos, A. E., Larsson, P. -L. and Vestergaard, R., Int. J. Solids Struct., 1994, 31, 2679.

19. Cheng, Y. T. and Cheng, C. M., J. Mater. Res., 1999, 14, 3493.

20. Giannakopoulos, A. E. and Suresh, S., Scripta mater., 1999, 40, 1191.

21. Venkatesh, T. A., Van Vliet, K. J., Giannakopoulos, A. E. and Suresh, S., Scripta mater., 2000, 42, 833.

22. Suresh, S. and Giannakopoulos, A. E., Acta mater., 1998, 46, 5755.

23. Bhattacharya, A. K. and Nix, W. D., Int. J. Solids Struct., 1988, 24, 881.

24. Laursen, T. A. and Simo, J. C., J. Mater. Res., 1992, 7, 618.

25. Tunvisut, K., O’Dowd, N. P. and Busso, E. P., Int. J. Solids Struct., 2001, 38, 335.

26. Hill, R., Storakers, B. and Zdunek, A. B., Proc. Roy. Soc. Lond. A, 1989, 423, 301.

27. Larsson, P. -L., Giannakopoulos, A. E., Soderlund, E., Rowcliffe, D. J. and Vestergaard, R., Int. J. Solids Struct., 1996, 33, 221.

28. Chaudhri, M. M., Acta mater., 1998, 46, 3047.

29. Johnson, K. L., Contact Mechanics. Cambridge University Press, London, 1985.

30. Fleck, N. A. and Hutchinson, J. W., J. Mech. Phys. Solids, 1993, 41, 1825.

31. Gao, H., Huang, Y., Nix, W. D. and Hutchinson, J. W., J. Mech. Phys. Solids, 1999, 47, 1239.

32. ABAQUS Theory Manual Version 6.1. Hibbitt, Karlsson and Sorensen Inc, Pawtucket, 2000.

33. King, R. B., Int. J. Solids Struct., 1987, 23, 1657.

34. MatWeb: http://www.matweb.com, 2001, by Automation Creations, Inc.

35. Ashby, M. F., Materials Selection in Mechanical Design. Butterworth Heinemann, Boston, 1999.

\section{APPENDIX A}

In this study, large deformation finite element computational simulations of depth-sensing indentation were carried out for 76 different combinations of elasto-plastic properties that encompass the wide range of parameters commonly found in pure and alloyed engineering metals; Young's modulus, $E$, was varied from 10 to $210 \mathrm{GPa}$, yield strength, $\sigma_{\mathrm{y}}$, from 30 to $3000 \mathrm{MPa}$, and strain hardening exponent, $n$, from 0 to 0.5 , and the Poisson's ratio, $v$, was fixed at 0.3 . Table A1 tabulates the elasto-plastic parameters used in these 76 cases.

\section{APPENDIX B}

In this appendix, six of the dimensionless functions identified in Section 3.1, i.e. $\Pi_{1}, \Pi_{2}, \Pi_{3}, \Pi_{4}, \Pi_{5}$ and $\Pi_{6}$, are listed explicitly. 
Table A1. Elasto-plastic parameters used in the present study

\begin{tabular}{llll}
\hline & $E(\mathrm{GPa})$ & $\sigma_{\mathrm{y}}(\mathrm{MPa})$ & $\sigma_{\mathrm{y}} / E$ \\
\hline 19 combinations of & & & \\
$E$ and $\sigma_{\mathrm{y}}{ }^{\mathrm{a}}$ & 10 & 30 & 0.003 \\
& 10 & 100 & 0.01 \\
10 & 300 & 0.03 \\
50 & 200 & 0.004 \\
50 & 600 & 0.012 \\
50 & 1000 & 0.02 \\
50 & 2000 & 0.04 \\
90 & 500 & 0.005556 \\
90 & 1500 & 0.016667 \\
90 & 3000 & 0.033333 \\
130 & 1000 & 0.007692 \\
130 & 2000 & 0.015385 \\
130 & 3000 & 0.023077 \\
170 & 300 & 0.001765 \\
170 & 1500 & 0.008824 \\
170 & 3000 & 0.017647 \\
210 & 300 & 0.001429 \\
210 & 1800 & 0.008571 \\
210 & 3000 & 0.014286 \\
\hline
\end{tabular}

${ }^{a}$ For each one of the 19 cases listed above, strain-hardening exponent $n$ is varied from $0,0.1,0.3$ to 0.5 , resulting a total of 76 different cases

$$
\begin{gathered}
\Pi_{1}=\frac{C}{\sigma_{0.033}}=-1.131\left[\ln \left(\frac{E^{*}}{\sigma_{0.033}}\right)\right]^{3} \\
+13.635\left[\ln \left(\frac{E^{*}}{\sigma_{0.033}}\right)\right]^{2} \\
-30.594\left[\ln \left(\frac{E^{*}}{\sigma_{0.033}}\right)\right]+29.267 \\
\Pi_{2}\left(\frac{E^{*}}{\sigma_{\mathrm{r}}}, n\right)=\frac{1 \mathrm{~d} P_{\mathrm{u}}}{E^{*} h_{\mathrm{m}} \mathrm{d} h}=\left(-1.40557 n^{3}\right. \\
+0.77526 n^{2}+0.15830 n \\
-0.06831)\left[\ln \left(\frac{E^{*}}{\sigma_{0.033}}\right)\right]^{3}+\left(17.93006 n^{3}\right. \\
-9.22091 n^{2}-2.37733 n \\
+0.86295)\left[\ln \left(\frac{E^{*}}{\sigma_{0.033}}\right)\right]^{2}+( \\
-79.99715 n^{3}+40.55620 n^{2}+9.00157 n
\end{gathered}
$$

$$
\begin{gathered}
-2.54543)\left[\ln \left(\frac{E^{*}}{\sigma_{0.033}}\right)\right]+\left(122.65069 n^{3}\right. \\
\left.-63.88418 n^{2}-9.58936 n+6.20045\right) \\
\Pi_{3}\left(\frac{\sigma_{r}}{E^{*}}, n\right)=\frac{h_{\mathrm{r}}}{h_{\mathrm{m}}}=\left(0.010100 n^{2}\right. \\
+0.0017639 n \\
-0.0040837)\left[\ln \left(\frac{\sigma_{0.033}}{E^{*}}\right)\right]^{3}+\left(0.14386 n^{2}\right. \\
+0.018153 n-0.088198)\left[\ln \left(\frac{\sigma_{0.033}}{E^{*}}\right)\right]^{2} \\
+\left(0.59505 n^{2}+0.034074 n\right. \\
-0.65417)\left[\ln \left(\frac{\sigma_{0.033}}{E^{*}}\right)\right]+\left(0.58180 n^{2}\right. \\
-0.088460 n-0.67290)
\end{gathered}
$$

$$
\begin{aligned}
\Pi_{4}=\frac{p_{\mathrm{ave}}}{E^{*}} \approx 0.268536(0.9952495 \\
\left.-\frac{h_{r}}{h_{m}}\right)^{1.1142735}
\end{aligned}
$$

$$
\begin{gathered}
\Pi_{5}=\frac{W_{\mathrm{p}}}{W_{\mathrm{t}}}=1.61217\{1.13111 \\
-1.74756\left[-1.49291\left(\frac{h_{\mathrm{r}}}{h_{\mathrm{m}}}\right)^{2.535334}\right] \\
\left.-0.075187\left(\frac{h_{\mathrm{r}}}{h_{\mathrm{m}}}\right)^{1.135826}\right\} \\
\Pi_{6}=\left.\frac{1 \mathrm{~d} P_{\mathrm{u}}}{E^{*} \sqrt{A_{\mathrm{m}}} \mathrm{d} h}\right|_{h_{\mathrm{m}}}=c^{*}
\end{gathered}
$$

